\title{
Model Categories and Simplicial Methods
}

\author{
Paul Goerss and Kristen Schemmerhorn
}

\begin{abstract}
There are many ways to present model categories, each with a different point of view. Here we'd like to treat model categories as a way to build and control resolutions. This an historical approach, as in his original and spectacular applications of model categories, Quillen used this technology as a way to construct resolutions in non-abelian settings; for example, in his work on the homology of commutative algebras [29], it was important to be very flexible with the notion of a free resolution of a commutative algebra. Similar issues arose in the paper on rational homotopy theory [31]. (This paper is the first place where the now-traditional axioms of a model category are enunciated.) We're going to emphasize the analog of projective resolutions, simply because these are the sort of resolutions most people see first. Of course, the theory is completely flexible and can work with injective resolutions as well.

There are now any number of excellent sources for getting into the subject and since this monograph is not intended to be complete, perhaps the reader should have some of these nearby. For example, the paper of Dwyer and Spalinski [16] is a superb and short introduction, and the books of Hovey [22] and Hirschhorn $[\mathbf{2 1}]$ provide much more in-depth analysis. For a focus on simplicial model categories - model categories enriched over simplicial sets in an appropriate way - one can read [20]. Reaching back a bit further, there's no harm in reading the classics, and Quillen's original monograph [30] certainly falls into that category.
\end{abstract}

\section{Contents}

1. Model Categories and Resolutions 2

2. Quillen Functors and Derived Functors 12

3. Generating New Model Categories 17

4. Simplicial Algebras and Resolutions in Non-abelian Settings 21

5. Resolutions in Model Categories 35

References $\quad 45$

2000 Mathematics Subject Classification. Primary: 18G55; Secondary: 18G10, 18G30, 18G50, 18G55, 55U35.

The first author was partially supported by the National Science Foundation. 


\section{Model Categories and Resolutions}

1.1. Chain complexes. Let us begin with a familiar and basic example. Nothing in this section is supposed to be new, except possibly the point of view.

Let $R$ be a commutative ring. Then we will denote the category of $R$-modules by $\operatorname{Mod}_{R}$. In this category, there is a distinguished class of objects in $\mathbf{M o d}$; namely, the projective $R$-modules. There is also a distinguished class of morphisms in $\operatorname{Mod}_{R}$, the surjections. These two classes determine each other; indeed, an $R$-module $P$ is projective if and only if

$$
\operatorname{Mod}_{R}(P, f): \operatorname{Mod}_{R}(P, M) \rightarrow \operatorname{Mod}_{R}(P, N)
$$

is a surjective map of sets for all surjections $f: M \rightarrow N$ of $R$-modules. Conversely, a morphism $f$ is a surjection if and only if $\operatorname{Mod}_{R}(P, f)$ is surjective for all projectives $P$.

There is a distinguished projective in $\operatorname{Mod}_{R}$, namely $R$ itself, and we can use that fact to show that $\operatorname{Mod}_{R}$ has enough projectives. This means that for all $M \in \operatorname{Mod}_{R}$, there is a surjection $P \rightarrow M$ with $P$ projective. (Here we combine the notions of a projective module and a surjective morphism.) If $M \in \mathbf{M o d}_{R}$, we define

$$
P(M)=\oplus_{f: R \rightarrow M} R
$$

where $f$ runs over the morphims in $\operatorname{Mod}_{R}$ from $R$ to $M$; then evaluation defines a surjection $\epsilon_{M}: P(M) \rightarrow M$. We have written this morphim as if it were a functor of $M$, and indeed it is. This will be useful later.

Now let $\mathbf{C h}_{*}(R)$ be the chain complexes in $\mathbf{M o d} \mathbf{d}_{R}$. By this we mean the non-negatively graded chain complexes, at least for now. These have the form

$$
\cdots \longrightarrow C_{3} \longrightarrow C_{2} \longrightarrow C_{1} \longrightarrow C_{0} \text {. }
$$

Then a projective resolution of a module $M \in \operatorname{Mod}_{R}$, is a chain complex $P_{\bullet}$ in $\mathbf{C h}_{*} R$, so that

(1) each $P_{n}$ is a projective;

(2) $H_{n} P_{\bullet}=0$ for $n>0$; and

(3) there is a morphism $P_{0}$ to $M$ which induces an isomorphim $H_{0} P_{\bullet} \cong M$.

We can rephrase the last two points. We regard $M$ as a chain complex concentrated in degree 0 and we can say that there is a morphism of chain complexes $P_{\bullet} \rightarrow M$ which induces an isomorphism on homology.

Chain complexes of projectives have the following important property. Recall that a chain complex $N_{\bullet}$ is acyclic if $H_{n} N_{\bullet}=0$ for $n>0$. Then there is automatically a homology isomorphism $N_{\bullet} \rightarrow H_{0} N_{\bullet}$, where the target is regarded as the chain complex concentrated in degree zero. Now suppose $P_{\bullet}$ is a chain complex so that each $P_{n}$ is projective; we are not assuming it is acyclic. Then, given any morphism of chain complexes $f: P_{\bullet} \rightarrow H_{0} N_{\bullet}$ (or, equivalently, a morphism $H_{0} P_{\bullet} \rightarrow H_{0} N_{\bullet}$ of modules) we can solve the lifting problem

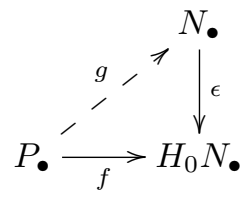


and $g$ is unique up to chain homotopy. This fact is then used to prove the uniqueness of projective resolutions up to chain homotopy.

It turns out that we can solve a much more general lifting problem than in (1.1). Here is the result.

Proposition 1.1. Suppose we have two morphisms $j: A_{\bullet} \rightarrow B_{\bullet}$ and $q: M_{\bullet} \rightarrow$



(1) for all $n \geq 0, A_{n} \rightarrow B_{n}$ is an injection and $B_{n} / A_{n}$ is projective; and

(2) $H_{*} q$ is an isomorphism and $M_{n} \longrightarrow N_{n}$ is a surjection for $n>0$.

Then any lifting problem

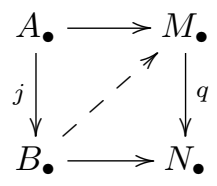

can be solved in such a way that both triangles commute.

We'll prove this below, but first let us record a lemma. The proof is a diagram chase. If $M_{\bullet}$ is a chain complex, let $Z_{n} M \subseteq M_{n}$ denote the cycles. We set $Z_{-1} M=$ 0 ; thus condition (2) in the following says $M_{0} \rightarrow N_{0}$ is onto.

Lemma 1.2. Let $f: M_{\bullet} \longrightarrow N_{\bullet}$ be a morphism of chain complexes. Then the following statements are equivalent:

(1) $H_{*} f$ is an isomorphism and $f: M_{n} \longrightarrow N_{n}$ is a surjection for $n>0$.

(2) The induced map

$$
M_{n} \longrightarrow Z_{n-1} M \times_{Z_{n-1} N} N_{n}
$$

is a surjection for $n \geq 0$.

Under either condition, the induced map $Z_{n} M \longrightarrow Z_{n} N$ is a surjection.

Proof of Proposition 1.1. We would like to construct the needed morphisms $g: B_{n} \rightarrow M_{n}$ by induction on $n$. For the inductive step we assume we have $g: B_{k} \rightarrow M_{k}$ for $k<n$ and that $g$ is a chain map as far as it is defined. Then we see that we need to solve a lifting problem

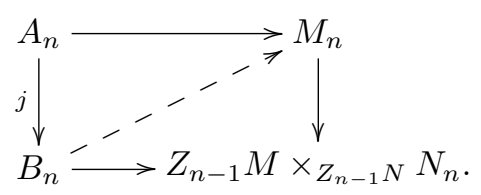

Now apply the previous lemma and the fact that $A_{n} \rightarrow B_{n}$ is isomorphic to a morphism of the form $A_{n} \rightarrow A_{n} \oplus P$, where $P$ is a projective.

1.2. Model Categories. The axioms for model categories are obtained by generalizing the example of chain complexes. We note that in $\mathbf{C h}_{*} R-$ and in particular in Proposition 1.1 - we identified three distinguished classes of maps: the homology isomorphisms, the morphisms $M_{\bullet} \rightarrow N_{\bullet}$ which were surjections in positive degrees, and the morphisms $A_{\bullet} \rightarrow B$ • which were injective with projective cokernel in all degrees. These will become, respectively, the weak equivalences, the fibrations, and the cofibrations for $\mathbf{C h}_{*} R$. As a matter of nomenclature, we say 
that a morphism $f: A \rightarrow B$ is a retract of a morphism $g: X \rightarrow Y$ is there is a commutative diagram

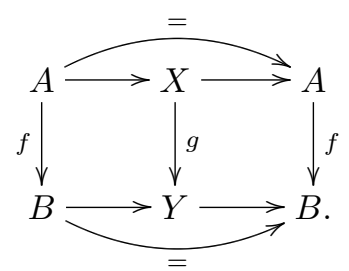

Definition 1.3. A model category is a category $\mathcal{C}$ with three classes of maps - weak equivalences, fibrations, and cofibrations - subject to the following axioms. An acyclic cofibration is a cofibration which is a weak equivalence. There is a corresponding notion of acyclic fibration.

M1. The category $\mathcal{C}$ is closed under limits and colimits;

M2. The three distinguished classes of maps are closed under retracts;

M3. (2 out of 3 ) Given $X \stackrel{f}{\longrightarrow} Y \stackrel{g}{\longrightarrow} Z$ so that any two of $f, g$, or $g f$ is a weak equivalence, then so is the third.

M4. (Lifting Axiom) Every lifting problem

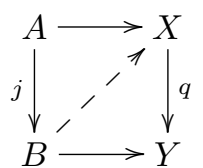

where $j$ is a cofibration and $q$ is a fibration has a solution so that both diagrams commute if one of $j$ or $q$ is a weak equivalence.

M5. (Factorization) Any $f: X \longrightarrow Y$ can be factored two ways:

(i) $X \stackrel{i}{\longrightarrow} Z \stackrel{q}{\longrightarrow} Y$, where $i$ is a cofibration and $q$ is a weak equivalence and a fibration.

(ii) $X \stackrel{j}{\longrightarrow} Z \stackrel{p}{\longrightarrow} Y$, where $j$ is a weak equivalence and a cofibration and $p$ is a fibration.

REMARK 1.4. We immediately make some of the standard comments about model categories.

(1) Note that CM4 and CM5 are really two axioms each. Also note that the axioms are completely symmetric in cofibrations and fibrations; thus the opposite category of $\mathcal{C}$ automatically inherits a model category structure.

(2) The three classes of maps are not independent. For example, if $\mathcal{C}$ is a model category, then the cofibrations are exactly the morphisms with the left lifting property (LLP) with respect to acyclic fibrations; that is, $j: A \rightarrow B$ is a cofibraton if and only if for every acyclic fibration $q: X \rightarrow Y$ and every lifting problem

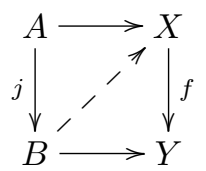

there is a solution so that both triangles commute. In an analogous fashion, a morphism is a fibration if and only if it has the RLP with respect to acyclic cofibrations. 
(3) Let $X$ be an object in the model category $\mathcal{C}$. Then $X$ is cofibrant if the unique morphism from the initial object to $X$ is a cofibration. More generally, a cofibrant replacement or cofibrant model for $X$ is a weak equivalence $Z \rightarrow X$ with $Z$ cofibrant. Such replacements always exist, by the factorization axiom, and we will discuss how unique such models are below when we talk about homotopies. (See Corollary 2.12.)

To be concrete, if $M \in \mathbf{M o d}_{R} \subseteq \mathbf{C h}_{*} R$ is an $R$-module regarded as a chain complex concentrated in degree 0 , then a cofibrant replacement for $M$ is simply a projective resolution.

There are corresponding notions of fibrant and fibrant replacement; for example, $X$ is fibrant if the unique morphism from $X$ to the terminal object is a fibration.

(4) In his original work on the subject, Quillen required a weakened version of M1; specifically, he only required finite limits and colimits. He had an example, as well: chain complexes of finitely generated $R$-modules.

(5) The class of cofibrations is closed under retracts and various colimits: coproducts, cobase change, and sequential colimits. For example, to be closed under cobase change means that if $i: A \rightarrow B$ is a cofibration and if we are given any push-out diagram

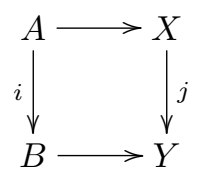

then $j$ is also a cofibration. Acyclic cofibrations are also closed under these operations, and there are similar closure properties for fibrations and acyclic fibrations - now using limits in place of colimits.

1.3. Chain complexes form a model category. The first example is chain complexes. For the record, we have:

TheOREM 1.5. The category $\mathbf{C h}_{*} R$ has the structure of a model category with a morphism $f: M_{\bullet} \rightarrow N_{\bullet}$

(1) a weak equivalence if $H_{*} f$ is an isomorphism;

(2) a fibration if $M_{n} \rightarrow N_{n}$ is surjective for $n \geq 1$; and,

(3) a cofibration if and only if for $n \geq 0$, the map $M_{n} \rightarrow N_{n}$ is an injection with projective cokernel.

We're going to prove this, as it gives us a chance to introduce some notation and terminology. First note that axioms M1, M2, and M3 are all completely straightforward. Also note that the "acyclic fibration" half of the lifting axiom M4 is exactly Proposition 1.1. The next step is to prove the "acyclic cofibration - fibration" half of the factorization axiom M5.

To do this, let $D(n), n \geq 1$ denote the chain complex with $D(n)_{k}=0$ for $k \neq n, n-1$ and

$$
\partial=1_{R}: D(n)_{n}=R \longrightarrow R=D(n)_{n-1} .
$$

Then there is a natural isomorphism $\mathbf{C h}_{*} R\left(D(n), N_{\bullet}\right) \cong N_{n}$ given by evaluation at the generator of $D(n)_{n}$. Therefore, $q: Q \bullet \rightarrow N_{\bullet}$ is a fibration if and only if $q$ has the the right lifting property with respect to the morphisms $0 \rightarrow D(n), n>0$. 
If $N_{\bullet}$ is any chain complex, define a new chain complex $P\left(N_{\bullet}\right)$ and an evaluation morphim $\epsilon: P\left(N_{\bullet}\right) \rightarrow N_{\bullet}$ by the equation

$$
P\left(N_{\bullet}\right)=\oplus_{n>0} \oplus_{x \in N_{n}} D(n) \longrightarrow N_{\bullet}
$$

This map is evidently a fibration. Better, if $M_{\bullet} \rightarrow N_{\bullet}$ is any morphism of chain complexes, we can extend this to a morphism

$$
M_{\bullet} \stackrel{j}{\longrightarrow} M_{\bullet} \oplus P\left(N_{\bullet}\right) \stackrel{q}{\longrightarrow} N_{\bullet}
$$

with $q$ a fibration and $j$ an acyclic cofibration with the left lifting property with respect to all fibrations. This completes this half of M5 and also allows us to prove the "acyclic cofibration" half of M4.

Indeed, if $i: M_{\bullet} \rightarrow N_{\bullet}$ is any acyclic cofibration, the factorization just completed yields a lifting problem

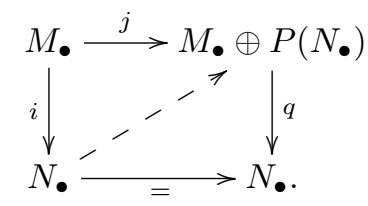

Since $i$ and $j$ are weak equivalences, so is the morphism $q$; hence, Proposition 1.1 supplies a solution to this lifting problem and any solution displays $i$ as a retract of $j$. Since $j$ has the left lifting property with respect to all fibrations, so does $i$. Thus lifting axiom M4 is verified.

This leaves the "cofibration-acyclic fibration" half of the factorization axion M5. We can prove this with an induction argument. Fix a morphism of chain complexes $f: M_{\bullet} \rightarrow N_{\bullet}$ and make the following induction hypothesis at $n \geq 0$ :

There are $R$-modules $Q_{k}, 0 \leq k \leq n-1$, and morphisms of $R$-modules $i$ : $M_{k} \rightarrow Q_{k}, p: Q_{k} \rightarrow N_{k}$ and $\partial: Q_{k} \rightarrow Q_{k-1}$ so that

(1) $p i=f: M_{k} \rightarrow N_{k}$;

(2) $\partial^{2}=0$ and $i$ and $p$ are chain maps as far as they are defined;

(3) $i$ is an injection with projective cokernel and

$$
Q_{k} \rightarrow Z_{k-1} Q \times{ }_{Z_{k-1} N} N_{k}
$$

is a surjection for $0 \leq k \leq n-1$.

Note that the case $n=0$ is true and easy, using the fact that all our chain complexes are zero in negative degrees. If we can complete the inductive step, Lemma 1.2 will complete M5 and the proof. But completing the inductive step uses the same idea we used at every other stage: that there are enough projectives. Indeed, the maps $i$ and $q$ give a morphism

$$
f^{\prime}: M_{n} \longrightarrow Z_{n-1} Q \times Z_{n-1} N N_{n}
$$

factoring $f$. Now choose a projective $P$ so that there is factoring of $f^{\prime}$

$$
M_{n} \longrightarrow M_{n} \oplus P \longrightarrow Z_{n-1} Q \times{ }_{Z_{n-1} N} N_{n}
$$

with the second morphism a surjection. Then set $Q_{n}=M_{n} \oplus P$.

As a final remark, note that with some care we could have made the factorizations natural. See Theorem 3.5. 
1.4. Other model categories. Here are some standard examples of model categories.

EXAMPLE 1.6. The category $\mathbf{C h}^{*} R$ of non-negatively graded cochain complexes of $R$-modules supports a model category structure which features the injective $R$-modules. The weak equivalences are $H^{*} f$ isomorphisms, the cofibrations are injections in positive degrees, and the fibrations are surjections with an injective kernel. Then the fibrant replacement of a constant cochain complex on an object $M$ is exactly an injective resolution. The argument proceeds exactly as in the previous section.

Less obvious is that there is a model category structure on $\mathbb{Z}$-graded chain complexes. The argument we used for non-negatively graded chain complexes used induction arguments which do not apply in this case. See $[\mathbf{2 2}] \S 2.3$. The weak equivalences are the homology isomorphisms, the fibrations the surjections, but cofibrations are slighly harder to understand: not every chain complex of projectives will be cofibrant. Note that this model category structure emphasizes projectives; there are other model category structures which focus on injectives.

EXAMPLE 1.7. Suppose $\mathcal{C}$ is a model category. Then there are some formal constructions we can make to create new model categories. For example, let $A \in \mathcal{C}$ be a fixed object and let $\mathcal{C} / A$ be the over category of $A$. Thus, the objects in $\mathcal{C} / A$ are arrows $X \rightarrow A$ and the morphisms are commutative triangles

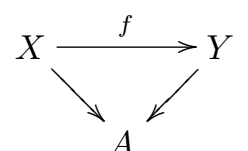

The morphism $f$ is a weak equivalence, fibration, or cofibration in $\mathcal{C} / A$ if it is so in $\mathcal{C}$. While this is easy, note that the fibrant objects have changed: $q: X \rightarrow A$ in $\mathcal{C} / A$ is fibrant if and only if $q$ is a fibration in $\mathcal{C}$.

EXAMPLE 1.8. Another basic example of a model category is the category of topological spaces, Top. A continuous map $f: X \longrightarrow Y$ is a weak equivalence if $f_{*}: \pi_{k}(X, v) \longrightarrow \pi_{k}(Y, f(v))$ is an isomorphism for $k \geq 0$ and all basepoints $v \in X{ }^{1}$ The fibrations are Serre fibrations and Example 3.2 below implies that $\mathrm{CW}$ complexes are cofibrant.

The category of all topological spaces has the disadvantage that it is not Cartesian closed: there is no exponential (or mapping space) functor right adjoint to the product. For this reason, we often restrict to the full subcategory CGH of compactly generated weak Hausdorff spaces. This will yield the same homotopy theory. (See the comments after Theorem 2.3.) The good categorical properties of compactly generated Hausdorff spaces are highlighted in the paper [33]. Unforunately, the best reference for the advantages of compactly generated weak Hausdorff spaces remains $[\mathbf{2 7}]$.

1.5. Simplicial Sets. One of the fundamental observations of non-abelian homological algebra is that the notion of a resolution of an object by a chain complex must be replaced by the notion of a resolution of an object by a simplicial object. In this section, we define simplicial objects and talk about the model category structure on simplicial sets.

\footnotetext{
$1^{1}$ This is the origin of the nomenclature "weak equivalence".
} 
For many years, the standard reference on simplicial sets has been $[\mathbf{2 8}]$, and this remains a good source. See also [8] and [17]. All of these references predate model structures, so for many years we got along quite well with [7], Chapter VIII. The reference $[\mathbf{2 0}]$ was written explicitly to work model categories into the mix.

Definition 1.9. The ordinal number category $\Delta$ has objects the ordered sets $[n]=\{0,1, \ldots, n\}$ and morphisms the (weakly) order preserving maps $\phi:[n] \longrightarrow$ $[m]$. In particular we have the maps

$$
\begin{aligned}
d^{i}:[n-1] & \longrightarrow[n], \quad 0 \leq i \leq n \\
\{0,1, \ldots, i-1, i, i+1, \ldots, n-1\} & \longmapsto\{0,1, \ldots, i-1, i+1, \ldots, n\}
\end{aligned}
$$

which skip $i$ and the maps

$$
\begin{aligned}
s^{j}:[n+1] & \longrightarrow[n] \text { for } 0 \leq j \leq n \\
\{0,1, \ldots, n+1\} & \longmapsto\{0,1, \ldots, j, j, \ldots, n\}
\end{aligned}
$$

which double up $j$. It is an exercise to show that all the morphisms in $\boldsymbol{\Delta}$ are compositions of these two types of morphisms. We also note that there are relations among them; for example,

$$
d^{i} d^{j}=d^{j} d^{i-1}, \quad i>j
$$

A full list of relations can be deduced from Lemma 1.11.

DEFINITION 1.10. A simplicial object in a category $\mathcal{C}$ is a contravariant functor from $\Delta$ to $\mathcal{C}$ :

$$
X: \boldsymbol{\Delta}^{\mathrm{op}} \longrightarrow \mathcal{C} .
$$

A morphism of simplicial objects is a natural transformation. The category of simplicial objects in $\mathcal{C}$ will be denoted $s \mathcal{C}$. As a matter of notation, we will write $X_{n}$ for $X([n])$,

$$
d_{i}=X\left(d^{i}\right): X_{n} \longrightarrow X_{n-1} \quad \text { and } \quad s_{i}=X\left(s^{i}\right): X_{n-1} \longrightarrow X_{n} .
$$

These are respectively, the face and degeneracy maps. More generally, if $\phi$ is morphism in $\Delta$, we will write $\phi^{*}$ for $X(\phi)$.

If $\mathcal{C}=$ Sets is the category of sets, we get the basic category $s$ Sets of simplicial sets.

The following is left as an exercise.

LEMMA 1.11. For any simplicial object $X \in s \mathcal{C}$, the face and degeneracy maps satisfy the following identities:

$$
\begin{aligned}
& d_{i} d_{j}=d_{j-1} d_{i} \quad i<j \\
& d_{i} s_{j}=s_{j-1} d_{i} \quad i<j \\
& =\quad 1 \quad i=j, j+1 \\
& =s_{j} d_{i-1} \quad i>j+1 \\
& s_{i} s_{j}=s_{j+1} s_{i} \quad i \leq j .
\end{aligned}
$$

Here are some examples of simplicial sets.

EXAMPLE 1.12 (The singular set). For $n \geq 0$, let $\sigma_{n}$ be the standard topological $n$-simplex; that is, the convex hull of the standard basis vectors in $\mathbb{R}^{n+1}$. If $\phi$ : $[n] \longrightarrow[m]$ is a morphism in $\boldsymbol{\Delta}$, then we have a map

$$
\phi_{*}: \sigma_{n} \longrightarrow \sigma_{m}
$$


dictated by $\phi$ on the vertices in their usual ordering and extended linearly to the rest of the simplex. Then we may define a functor

$$
S(-): \text { Top } \rightarrow s \text { Sets }
$$

by setting $S_{n}(X)=\operatorname{Top}\left(\sigma_{n}, X\right)$ and $\phi^{*}=\operatorname{Top}\left(\phi_{*}, X\right)$. This is the singular set of $X$.

This object is quite familiar: if we apply the free abelian group functor levelwise to $S(X)$ and take the alternating sum of the face maps, we obtain the singular chain complex of first-year algebraic topology.

Definition 1.13. If $X$ is a simplicial set, we call $X_{n}$ the $n$-simplices and the subset

$$
\bigcup_{i=0}^{n-1} s_{i} X_{n-1} \subseteq X_{n}
$$

the degenerate simplices. Note that the degenerate simplices could equally be written as

$$
\bigcup_{\phi:[n] \rightarrow[k]} \phi^{*} X_{k} \subseteq X_{n}
$$

where $\phi$ runs over the non-identity surjections out of $[n]$ in $\Delta$.

EXAMPLE 1.14. Let $K$ be an ordered simplicial complex; that is, a simplicial complex with an ordering on the set $V$ of vertices $K$. Define a simplicial set $K$. with $K_{n}$ the set of collections

$$
v_{0} \leq v_{1} \leq v_{2} \leq \ldots \leq v_{n}
$$

of vertices subject to the requirement that the $v_{i}$, after eliminating repetitions, are the vertices of a simplex of $K$. Thus, for example, if $K$ is the boundary of the standard 2-simplex with vertices $e_{i}$, then $e_{0} \leq e_{1} \leq e_{1}$ is in $K_{2}$, but $e_{0} \leq e_{1} \leq e_{2}$ is not. The face and degeneracy operators are defined by doubling or deleting one of the $v_{i}$.

EXAMPLE 1.15 (The standard simplices). The functor from simplicial sets to sets sending $X$ to $X_{n}$ is representable, by the Yoneda Lemma. Indeed, define the standard $n$-simplex $\Delta^{n}$ of $s$ Sets by

$$
\Delta^{n}=\Delta(-,[n]): \Delta^{o p} \rightarrow \text { Sets. }
$$

Then the Yoneda Lemma supplies the isomorphism $s \operatorname{Sets}\left(\Delta^{n}, X\right) \cong X_{n}$. The morphisms in $\Delta$ yield morphisms $\Delta^{m} \rightarrow \Delta^{n}$.

We also have the boundary of the standard simplex

$$
\partial \Delta^{n}=\bigcup_{0 \leq i \leq n} d^{i} \Delta^{n-1} \subseteq \Delta^{n}
$$

and the horns

$$
\Delta_{k}^{n}=\bigcup_{i \neq k} d^{i} \Delta^{n-1} \subseteq \Delta^{n} .
$$

All of these simplicial sets can also be realized by the construction of Example 1.14 using some appropriate subcomplex of the standard topological $n$-simplex $\sigma_{n}$. 
EXAMPLE 1.16 (The nerve of a small category). Let $\mathcal{I}$ be a small category; that is, a category with a set of objects. Then we define a simplicial set $B \mathcal{I}-$ the nerve of the category - with the $n$-simplices all strings of composable arrows in $\mathcal{I}$ :

$$
B \mathcal{I}_{n}=\left\{x_{0} \rightarrow x_{1} \rightarrow \cdots \rightarrow x_{n}\right\} .
$$

If $\phi:[m] \rightarrow[n]$ is a morphism in $\Delta$, then the induced function $\phi^{*}: B \mathcal{I}_{n} \rightarrow B \mathcal{I}_{m}$ is given by

$$
\phi^{*}\left(\left\{x_{0} \rightarrow x_{1} \rightarrow \cdots \rightarrow x_{n}\right\}\right)=\left\{x_{\phi(0)} \rightarrow x_{\phi(1)} \rightarrow \cdots \rightarrow x_{\phi(m)}\right\}
$$

where we use composition, deletion, or insertion of identities as necessary. For example, the three face maps from $B \mathcal{I}_{2}$ to $B \mathcal{I}_{1}$ are given by

$$
\begin{array}{rll}
\left\{x_{0} \rightarrow x_{1} \rightarrow x_{2}\right\} & \stackrel{d_{0}}{\longmapsto} & \left\{x_{1} \rightarrow x_{2}\right\} \\
& \stackrel{d_{1}}{\longmapsto} & \left\{x_{0} \rightarrow x_{2}\right\} \\
& \stackrel{d_{2}}{\longmapsto} & \left\{x_{0} \rightarrow x_{1}\right\} .
\end{array}
$$

To be concrete, if $\mathcal{I}$ is the category

$$
0 \rightarrow 1 \rightarrow \cdots \rightarrow n
$$

then $B \mathcal{I} \cong \Delta^{n}$.

EXAMPLE 1.17. To make your confusion completely specific ${ }^{2}$, consider $\Delta^{1}$ as the nerve of $0 \rightarrow 1$. Then we have

$$
\Delta_{0}^{1}=\{0,1\}
$$

and

and

$$
\boldsymbol{\Delta}_{1}^{1}=\{0 \rightarrow 0,0 \rightarrow 1,1 \rightarrow 1\}
$$

$$
\boldsymbol{\Delta}_{2}^{1}=\{0 \rightarrow 0 \rightarrow 0,0 \rightarrow 0 \rightarrow 1,0 \rightarrow 1 \rightarrow 1\} .
$$

Thus the only non-degenerate simplex in $\boldsymbol{\Delta}_{1}^{1}$ is $0 \rightarrow 1$ and all of the simplices in $\boldsymbol{\Delta}_{2}^{1}$ are degenerate.

EXAMPLE 1.18 (The product of simplicial sets). Unlike the product of simplicial complexes, the product of simplicial sets is easy to describe. If $X$ and $Y$ are simplicial sets, then $(X \times Y)_{n}=X_{n} \times Y_{n}$ and $\phi$ is a morphism in $\Delta$, then

$$
\phi_{(X \times Y)}^{*}=\phi_{X}^{*} \times \phi_{Y}^{*} \text {. }
$$

Definition 1.19. For a simplicial set $X$ define a topological space $|X|$ - the geometric realization of $X$ - by taking a coequalizer in the category CGH of compactly generated weak Hausdorff spaces (see Example 1.8):

$$
\coprod_{\phi:[n] \rightarrow[m]} X_{m} \times \sigma_{n} \longrightarrow \coprod_{n} X_{n} \times \sigma_{n} \longrightarrow|X|
$$

Here $\sigma_{n}$ is the topological $n$-simplex and $X_{n} \times \sigma_{n}=\coprod_{X_{n}} \sigma_{n}$. The parallel arrows are the two arrows that are induced by evaluating on $X$ or on $\sigma_{(-)}$respectively.

The following result tabulates some facts about the geometric realization functor. The singular set functor was defined in Example 1.12. In the second point we are using the product in CGH - the "Kelly product". See [33].

\footnotetext{
${ }^{2}$ This joke is stolen from Steve Wilson.
} 
Proposition 1.20. The geometric realization functor has the following properties:

(1) The functor $|-|: s$ Sets $\rightarrow \mathbf{C G H}$ is left adjoint to the singular set functor $S(-)$.

(2) If $X$ and $Y$ are simplicial sets, then the natural morphism $|X \times Y| \rightarrow$ $|X| \times|Y|$ in $\mathbf{C G H}$ is a homeomorphism.

(3) If $\Delta^{n}$ is the simplicial n-simplex, then $\left|\Delta^{n}\right| \cong \sigma_{n}$.

REMARK 1.21. We use geometric realization to depict simplicial sets. Only the non-degenerate simplices are needed for a picture of $|X|$, and the face maps tell us how to glue them together. For example, for $\boldsymbol{\Delta}^{1} \times \boldsymbol{\Delta}^{1}$ we have the following picture of the geometric realization:

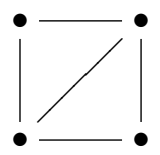

There are four non-degenerate 0 -simplices, five non-degenerate 1 -simplices and two non-degenerate 2 -simplices glued as shown. (There are also orientations on the 1and 2-simplices, which are not shown.) Note that while $\Delta^{1}$ has no non-degenerate 2 -simplices, $\Delta^{1} \times \Delta^{1}$ indeed has two non-degenerate 2 -simplices. It is an extremely useful exercise to identify these elements of $\left(\Delta^{1} \times \Delta^{1}\right)_{2}$.

We now come to the model category structure on simplicial sets. It is still one of the deeper results in the theory. I know of no easy proof, nor of one that doesn't essentially also prove the Quillen equivalence of simplicial sets and topological spaces at the same time. (See Theorem 2.3 below.) The arguments are spelled out in $[\mathbf{2 0}]$ and $[\mathbf{2 2}]$ but these are variations on Quillen's original argument of [30].

THEOREM 1.22. The category sSets has the structure of a model category with a morphism $f: X \rightarrow Y$

(1) a weak equivalence if $|f|:|X| \rightarrow|Y|$ is a weak equivalence of topological spaces;

(2) a cofibration if $f_{n}: X_{n} \rightarrow Y_{n}$ is a monomorphism for all $n$; and

(3) a fibration if $f$ has the left lifting property with respect to the inclusions of the horns

$$
\Delta_{k}^{n} \longrightarrow \Delta^{n}, \quad n \geq 1,0 \leq k \leq n .
$$

The lifting condition in the definition of fibration for simplicial sets is known as the Kan extension condition and goes back to Kan's original work on the subject in [26]. It arose there as a condition that made it possible to define and analyze the homotopy groups of a simplicial set without referring to topological spaces. (Compare Example 2.15.) However, the lifting condition implies that a fibrant simplicial set has very many simplices, which makes them hard to draw: for example, $\Delta^{n}$ is not fibrant if $n>0$. Or, for another example, if $\mathcal{I}$ is a small category, its nerve $B \mathcal{I}$ is fibrant if and only if $\mathcal{I}$ is a groupoid.

For some good news, however, we note that every simplicial group, regarded as a simplicial set, is fibrant; indeed, every surjective morphism of simplicial groups is a fibration. To make a definitive statement along these lines, let us make a 
definition. If $X$ is a simplicial set, define $\pi_{0} X$ by the coequalizer diagram of sets

$$
X_{1} \underset{d_{0}}{\stackrel{d_{1}}{\longrightarrow}} X_{0} \longrightarrow \pi_{0} X \text {. }
$$

It is an exercise to show that $\pi_{0} X \cong \pi_{0}|X|$. The following is also left to the reader.

Proposition 1.23. Let $f: X \rightarrow Y$ be a morphism of simplicial groups. Then $f$, regarded as a morphism of simplicial sets, is a fibration if and only if

$$
X \longrightarrow \pi_{0} X \times_{\pi_{0} Y} Y
$$

is a surjection.

\section{Quillen Functors and Derived Functors}

We have defined the objects of interest - model categories - and we will now define and study the morphisms between them, which are Quillen functors. Then we will introduce the notion of the homotopy category of a model category and discuss the fact that Quillen functors descend to adjoint functors between the homotopy categories. This is a little backwards - traditionally one defined the homotopy category almost immediately after defining the model category - but the lesson of the last thirty or so years is that there's more information in the model category than in the homotopy category and we should descend later rather than sooner.

2.1. Quillen Functors and Quillen Equivalence. Here are our morphisms between model categories.

Definition 2.1. Let $\mathcal{C}$ and $\mathcal{D}$ be two model categories. Then a Quillen functor from $\mathcal{C}$ to $\mathcal{D}$ is an adjoint pair of functors

$$
\mathcal{C} \underset{G}{\stackrel{F}{\rightleftarrows}} \mathcal{D}
$$

with $F$ the left adjoint so that

(1) the functor $F$ preserves cofibrations and weak equivalences between cofibrant objects, and

(2) the functor $G$ preserves fibrations and weak equivalences between fibrant objects.

A Quillen functor is a Quillen equivalence if for all cofibrant objects $X$ in $\mathcal{C}$ and all fibrant objects $Y$ in $\mathcal{D}$, a morphism

$$
X \longrightarrow G Y
$$

is a weak equivalence in $\mathcal{C}$ if and only if the adjoint morphism

$$
F X \longrightarrow Y
$$

is a weak equivalence in $\mathcal{D}$.

Here and elsewhere we will write an adjoint pair of functors with the left adjoint on top, and we regard this as a "function" from $\mathcal{C}$ to $\mathcal{D}$.

EXAMPle 2.2. Let $f: R \longrightarrow S$ be a homomorphism of commutative rings and let $\operatorname{res}_{f}$ denote the restriction of scalars functor from $S$-modules to $R$-modules.

$$
S \otimes_{R}-: \mathbf{C h}_{*} R \longrightarrow \mathbf{C h}_{*} S: \operatorname{res}_{f}
$$


yields a Quillen functor. Note that the functor res $_{f}$ preserves all weak equivalences. This is a Quillen equivalence only if $R=S$.

The following liberating result, which first appeared in [30], has its roots in the work of Kan and is a modern formulation of Kan's observation that the homotopy theory of topological spaces and the homotopy theory of simplicial sets are equivalent.

THEOREM 2.3. The geometric realization functor and the singular set functor give a Quillen equivalence

$$
|-|: s \text { Sets } \longleftrightarrow \text { CGH }: S(-) .
$$

One might quibble that one would really want the homotopy theory of all topological spaces, not only compactly generated weak Hausdorff spaces. However, a result we leave the reader to formulate yields an appropriate Quillen equivalence between the two. But we should also append the remark that we are using the Serre model category structure on spaces, as in Example 1.8. There is another model category structure on spaces wherein the weak equivalences are actually homotopy equivalences. See [35]. This is another story all together, and we leave it for another day.

2.2. Homotopies and the homotopy category. So far we have been writing about model categories as if they encoded a homotopy theory. This is indeed the case, and in this section, we make this precise.

Definition 2.4. Let $A \in \mathcal{C}$, where $\mathcal{C}$ is a model category. A cylinder object for $A$ is a factoring



where $i$ is a cofibration, $q$ is a weak equivalence, and $\nabla$ is the fold map. We will refer to $C(A)$ as the cylinder object, leaving the rest of the diagram implicit.

Example 2.5. If $A$ is a $\mathrm{CW}$ complex, then we may choose $C(A)=A \times[0,1]$. But note that if $A$ is not cofibrant as a topological space, than the inclusion

$$
A \amalg A \longrightarrow A \times[0,1]
$$

may not be a cofibration. We also remark that at this point that this is an example of a natural cylinder object: this is desirable, and may even be required at certain points. Compare Theorem 3.8.

EXAMPLE 2.6. We may define a natural cylinder object for chain complexes as follows. Let $P_{\bullet} \in \mathbf{C h}_{*} R$ be cofibrant, and set

$$
C\left(P_{\bullet}\right)_{n}=P_{n} \oplus P_{n-1} \oplus P_{n}
$$

with boundary

$$
\partial(x, a, y)=(\partial x+a,-\partial a, \partial y+a) .
$$

The following definition is motivated by the example of topological spaces. 
Definition 2.7. Let $f, g: A \longrightarrow X$ be two morphisms in a model category $\mathcal{C}$. A left homotopy from $f$ to $g$ is a diagram in $\mathcal{C}$

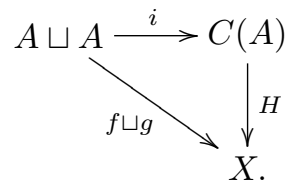

where $C(A)$ is a cylinder object for $A$. We will denote the homotopy by $H$ and drop the "left" when it can be understood from the context.

REMARK 2.8. We leave it to the reader to formulate the appropriate notions of a path object and right homotopy. These ideas are completely dual to those just presented.

EXAMPLE 2.9. Using the cylinder object for chain complexes we wrote down in Example 2.6 we see that in $\mathbf{C h}_{*} R$, the homotopies correspond to the chain homotopies.

ExAmple 2.10. Here is a formal example. Given $f: A \rightarrow X$, the constant homotopy is given by the diagram

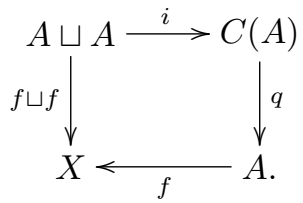

If $\varphi: X \rightarrow Y$ is a morphism in $\mathcal{C}$ and $f, g: A \rightarrow X$ are two morphisms so that $\varphi f=\varphi g$, then a homotopy from $f$ to $g$ over $Y$ is a homotopy $H: C(A) \rightarrow X$ so that $\varphi H$ is the constant homotopy. There is also the notion of a right homotopy under an object.

The following result now follows from the model category axioms.

LEMMA 2.11. Suppose there is a lifting problem in a model category $\mathcal{C}$



where $j$ is a cofibration, $q$ is a fibration, and one of $j$ or $q$ is a weak equivalence. Then $f$ exists and $f$ is unique up to left homotopy under $A$ and over $Y$. In other words, any two solutions to the lifting problem are homotopic via a homotopy which reduces to the constant homotopy when restricted to $A$ or pushed forward to $Y$.

The next result now follows immediately and provides a uniqueness result for cofibrant replacements. Note that the model category axioms imply that for any object $A$ in a model category there is an acyclic fibration $X \rightarrow A$ with $X$ cofibrant.

Corollary 2.12. Let $\mathcal{C}$ be a model category and $A$ an object in $\mathcal{C}$. Suppose we are given a weak equivalence $f_{1}: X_{1} \rightarrow A$ and an acyclic fibration $f_{2}: X_{2} \rightarrow A$ with $X_{1}$ and $X_{2}$ cofibrant. Then there is a weak equivalence $X_{1} \rightarrow X_{2}$ over $A$ and this morphism is unique up to homotopy over $A$. Furthermore, if $f_{1}$ is an acyclic fibration, then $X_{1}$ and $X_{2}$ are homotopy equivalent over $A$. 
As before we leave the reader to formulate a version of this result for fibrant replacements.

Definition 2.13 (The homotopy category). Let $\mathcal{C}$ be a model category. Then the homotopy category $\operatorname{Ho}(\mathcal{C})$ is the category obtained from $\mathcal{C}$ by inverting the weak equivalences. Thus $\operatorname{Ho}(\mathcal{C})$ is characterized by the property that there is a functor $\iota: \mathcal{C} \rightarrow \mathbf{H o}(\mathcal{C})$ which takes weak equivalences to isomorphisms and if $F: \mathcal{C} \rightarrow \mathcal{D}$ is any functor taking weak equivalences to isomorphisms then there is a unique functor $F^{\prime}: \mathbf{H o}(\mathcal{C}) \rightarrow \mathcal{D}$ making the following diagram commute



We may write $\mathbf{H o}(\mathcal{C})(X, Y)$ as $[X, Y]_{\mathcal{C}}$.

The morphisms in $\operatorname{Ho}(\mathcal{C})$ can be described as zig-zags where the reverse arrows are weak equivalences, but this begs the question of whether the morphism sets are actually sets. It is more practical to give the following two descriptions of the morphisms. First, there is an isomorphism

$$
\operatorname{Ho}(\mathcal{C})(X, Y) \cong \mathcal{C}\left(X_{c}, Y_{f}\right) /(\sim \text { homotopy }),
$$

where $X_{c} \rightarrow X$ is a cofibrant replacement and $Y \rightarrow Y_{f}$ is fibrant replacement. The fact that the right hand side of this equation is well-defined follows from Corollary 2.12 and its fibrant analog.

Second, the following result, due to Dwyer and Kan [11], gives a simple choicefree description of the morphisms, although it also has its set-theoretic issues.

Lemma 2.14. Let $\mathcal{C}$ be a model category and let $X$ and $Y$ be objects in $\mathcal{C}$. Then $\operatorname{Ho}(\mathcal{C})(X, Y)$ is isomorphic to the set of equivalence classes of diagrams in $\mathcal{C}$

$$
X \stackrel{\simeq}{\longleftarrow} \longrightarrow V \stackrel{\simeq}{\longleftarrow} Y
$$

where the left-facing arrows are weak equivalences. The equivalence relation among these diagrams is the smallest equivalence relation containing the relation created by diagrams of the form<smiles>[Y][Al]1[Y]2[Y][Y1]21[Y]</smiles>

As the reader may have surmised, this description of the morphisms in the homotopy category can be obtained by taking the components of the nerve of a suitable category.

EXAMPLE 2.15 (Homotopy groups of simplicial sets). In simplicial sets, we can take $\Delta^{n} / \partial \Delta^{n}$ as a model for the $n$-sphere. Hence, if $X$ is a pointed fibrant simplicial set, $\pi_{n} X$ can be calculated as the based homotopy classes of maps $\Delta^{n} / \partial \Delta^{n} \rightarrow X$. Since the geometric realization of $\Delta^{n} / \partial \Delta^{n}$ is the topological $n$-sphere, Theorem 2.3 implies that these homotopy groups are naturally isomorphic to the homotopy groups of the geometric realization of $X$. This yields Kan's combinatorial definition of homotopy groups. See $[\mathbf{2 6}]$. 
If $X$ is a simplicial group, then $X$ is automatically fibrant and there is a natural isomorphism

$$
s \operatorname{Sets}_{*}\left(\Delta^{n} / \partial \Delta^{n}, X\right) \cong \bigcap_{i=0}^{n} \operatorname{Ker}\left\{d_{i}: X_{n} \longrightarrow X_{n-1}\right\}
$$

(Here the asterisk means were are taking based maps; $e \in X$ is the basepoint of the simplicial group $X$.) Furthermore, a morphism $\Delta^{n} / \partial \Delta^{n} \rightarrow X$ is null-homotopic if and only if it can be factored

$$
\Delta^{n} / \partial \Delta^{n} \stackrel{d^{0}}{\longrightarrow} \Delta^{n+1} / \Delta_{0}^{n+1} \longrightarrow X
$$

Hence, if we set

$$
N X_{n}=\bigcap_{i=1}^{n} \operatorname{Ker}\left\{d_{i}: X_{n} \longrightarrow X_{n-1}\right\}
$$

the homomorphisms $d_{0}: N X_{n+1} \rightarrow N X_{n}$ satisfy $d_{0}^{2}=0$ and we can conclude that $\pi_{n} X \cong H_{n}(N X)$. The chain complex of not-necessarily abelian groups $\left(N X, d_{0}\right)$ is called the Moore complex. If $X$ is a simplicial abelian group, $N X$ is also called the normalized chain complex of $X$. See $\S 4.1$ for more discussion.

2.3. Total derived functors. Returning to the thread of model categories as a source of resolutions, we now use model categories to define derived functors.

Definition 2.16. Let $F: \mathcal{C} \rightleftarrows \mathcal{D}: G$ be a Quillen functor. Then $F$ has a total left derived functor $L F$ defined as follows. If $X \in \mathcal{C}$, let $X_{c} \rightarrow X$ be a weak equivalence with $X_{c}$ cofibrant. Then set $L F(X)=F\left(X_{c}\right)$.

It immediately follows from Corollary 2.12 that $L F(X)$ is independent, up to weak equivalence, of the choice of cofibrant replacement, and is well-defined in the homotopy category of $\mathcal{D}$. As a side remark, we can weaken the assumption that we have a Quillen functor. Quillen, for example, describes the total left derived functor as a Kan extension of sorts. See [30] or [20] $\S I I .7$.

Here is a first example. There will be others below.

EXAMPLE 2.17. Let $f: R \rightarrow S$ be a morphism of commutative rings and consider the Quillen functor of Example 2.2

$$
S \otimes_{R}-: \mathbf{C h}_{*} R \rightleftarrows \mathbf{C h}_{*} S: \operatorname{res}_{f} .
$$

Let $M \in \operatorname{Mod}_{R} \subseteq \mathbf{C h}^{*} R$ and $P . \stackrel{\cong}{\longrightarrow} M$ be a projective resolution (i.e., a cofibrant replacement) of $\bar{M}$. Then

$$
L\left(S \otimes_{R}-\right)(M)=S \otimes_{R} P_{\bullet}
$$

In particular,

$$
H_{n} L\left(S \otimes_{R}-\right)(M)=\operatorname{Tor}_{n}^{R}(S, M) .
$$

For more general objects $N_{\bullet} \in \mathbf{C h}_{*} R$, there is a spectral sequence

$$
\operatorname{Tor}_{p}^{R}\left(S, H_{q} N_{\bullet}\right) \Longrightarrow H_{p+q} L\left(S \otimes_{R}-\right)\left(N_{\bullet}\right) .
$$

See [30], §II.6 or Example 5.19. Note that we often write

$$
L\left(S \otimes_{R}-\right)\left(N_{\bullet}\right)=S \otimes_{R}^{L} N_{\bullet}
$$


There is also a companion notion of total right derived functor. Quillen introduced the notion of a Quillen functor (without using that name, of course) in order to prove the following result - which applies, in particular, to the case of simplicial sets and topological spaces. See Theorem 2.3.

Proposition 2.18. Let $F: \mathcal{C} \rightleftarrows \mathcal{D}: G$ be a Quillen functor between two model categories. Then the total derived functors induce an adjoint pair

$$
L F: \mathbf{H o}(\mathcal{C}) \longleftrightarrow \mathbf{H o}(\mathcal{D}): R G .
$$

Furthermore, this adjoint pair induces an equivalence of categories if and only if the Quillen functor is a Quillen equivalence.

\section{Generating New Model Categories}

In this section we discuss how to promote model category structures from one category to another. The basic idea is to take an adjoint pair $F: \mathcal{C} \rightleftarrows \mathcal{D}: G$ and a model category structure on $\mathcal{C}$, and then force it to be a Quillen functor. To do this requires structure and hypotheses, which we explore first.

\subsection{Cofibrantly Generated Model Categories.}

Definition 3.1. Let $\mathcal{C}$ be a category and let $\mathcal{F} \subseteq \mathcal{C}$ be a class of maps. Then $A \in \mathcal{C}$ is small for $\mathcal{F}$ if whenever

$$
X_{1} \rightarrow X_{2} \rightarrow X_{3} \rightarrow \cdots
$$

is a sequence of morphisms in $\mathcal{F}$, then the natural map

$$
\operatorname{colim} \mathcal{C}\left(A, X_{n}\right) \longrightarrow \mathcal{C}\left(A, \operatorname{colim} X_{n}\right)
$$

is an isomorphism.

Here we are taking colimits over the natural numbers. Once could define a notion of $\lambda$-small where $\lambda$ is any ordinal number. This is useful for localization theory (see [21]), but won't appear in any of our examples here.

EXAMPLES 3.2. Here are some basic examples.

(1) Any compact space $A$ is $J$-small, when $J$ is the class of closed inclusions.

(2) Any bounded chain complex of finitely presented $R$-modules is $J$-small, for $J$ all morphisms of chain complexes.

(3) Any finite simplicial set $X$ - meaning $X$ has finitely many non-degenerate simplices - is $J$-small, for $J$ the class of all morphisms of simplicial sets. This is one of the reasons that simplicial sets are technically pleasant.

Definition 3.3. A model category is cofibrantly generated if there are sets of morphisms $I$ and $J$ so that

(1) the source of every morphism in $I$ is small for the class of all cofibrations and $q: X \longrightarrow Y$ is an acyclic fibration if and only if $q$ has the RLP with respect to all morphisms of $I$; and

(2) the source of every morphism in $J$ is small with respect to the class of all acyclic cofibrations and $q: X \longrightarrow Y$ is a fibration if and only if $q$ has the RLP with respect to all morphisms of $J$. 
The set $I$ and the set $J$ generate the cofibrations and the acyclic cofibrations repsectively. Here, "generate" means the cofibrations are the smallest class of maps that contains $I$ and is closed under coproducts, cobase change, sequential colimits, and retracts.

EXAMPLES 3.4. All of our basic model categories are cofibrantly generated.

1. First consider the category $\mathbf{C h}_{*} R$ of chain complexes over a commutative ring $R$. The set $J$ of generating acyclic cofibrations can be taken to be the morphisms

$$
0 \longrightarrow D(n), \quad n \geq 1
$$

where $D(n)$ is the object so that $\mathbf{C h}_{*} R\left(D(n), M_{\bullet}\right) \cong M_{n}$. (See Equation 1.2.) To specify a set of generating cofibrations, let $S(n)$ be the chain complex with $S(n)_{n}=R$ and $S(n)_{k}=0$ for $k \neq n$. Then the set $I$ can be chosen to consist of the obvious inclusions

$$
\begin{aligned}
S(n-1) & \longrightarrow D(n), \quad n \geq 1 ; \\
0 & \longrightarrow S(0)
\end{aligned}
$$

This follows immediately from Proposition 1.1.

2. The Serre model category on compactly generated weak Hausdorff spaces is cofibrantly generated. We may choose the set $I$ of generating cofibrations to be inclusions of boundaries of the disks

$$
S^{n-1}=\partial D^{n} \longrightarrow D^{n}, \quad n \geq 0
$$

where $S^{-1}=\emptyset$ and we may choose $J$ to be the inclusions

$$
D^{n} \longrightarrow D^{n} \times[0,1], \quad n \geq 0 .
$$

Indeed, to have the RLP with respect to the elements of $J$ is exactly the definition of a Serre fibration.

3. The category of simplicial sets is cofibrantly generated. The generating cofibrations can be chosen to be the inclusions

$$
\partial \Delta^{n} \longrightarrow \Delta^{n}, \quad n \geq 0
$$

where, again $\partial \Delta^{0}=\emptyset$ and the generating acyclic cofibrations are the inclusions of the horns ${ }^{3}$

$$
\Delta_{k}^{n} \longrightarrow \Delta^{n}, \quad 0 \leq k \leq n, n \geq 1 .
$$

The horns and $\partial \Delta^{n}$ were defined in Example 1.15.

4. But not every model category is cofibrantly generated. Perhaps the easiest example, admittedly a bit artificial, is the induced model category structure on the opposite category of simplicial sets.

We now come to a basic fact about cofibrantly generated model categories. The proof of this fact may be more important than the result itself. Called Quillen's small object argument, it first appeared in [30], §II.4, and we present it in some detail.

THEOREM 3.5 (The small object argument). If $\mathcal{C}$ is cofibrantly generated, then factorizations of M5 can be chosen to be natural.

\footnotetext{
${ }^{3}$ Also known as anodyne extensions. See [17].
} 
Proof. We will do the "cofibration-acylic fibration" factorization; the other is similar. Let $f: X \longrightarrow Y$ in $\mathcal{C}$. We want to factor $f$ in a natural way as

$$
X \stackrel{j}{\longrightarrow} Z \stackrel{q}{\longrightarrow} Y
$$

where $j$ is a cofibration and $q$ is an acyclic fibration. To do this, we produce the diagram over the object $Y$

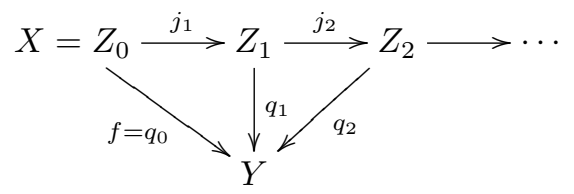

so that there is a pushout diagram



where $U$ is the set of diagrams of the following form



with $i \in I$. Then $Z=\operatorname{colim} Z_{n}$ and $q=\operatorname{colim} q_{n}$. The induced map $j: X \rightarrow Z$ is a cofibration because it is generated naturally by elements in $I$. Thus we need only show that $q$ is an acyclic fibration. For this, it is sufficient to find a solution to every lifting problem

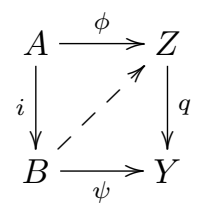

where $i \in I$. Because $A$ is small with respect to the class of all cofibrations, there is a factoring



for some $n$; therefore, by the construction of $Z_{n+1}$, we have a diagram

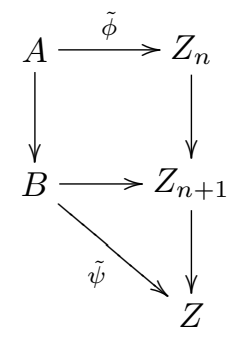


and $\tilde{\psi}$ solves the original lifting problem.

3.2. Promoting model category structures. We now give a result - again essentially due to Quillen - for lifting a model category structure from one category to another. Recall from Remark 1.4.2 that once we have specified weak equivalences and fibrations, then cofibrations will be forced.

THEOREM 3.6. Let $F: \mathcal{C} \longleftrightarrow \mathcal{D}: G$ be an adjoint pair and suppose $\mathcal{C}$ is a cofibrantly generated model category. Let $I$ and $J$ be chosen sets of generating cofibrations and acyclic cofibrations, respectively. Define a morphism $f: X \rightarrow Y$ in $\mathcal{D}$ to be a weak equivalence or a fibration if $G f$ is a weak equivalence or fibration in $\mathcal{C}$. Suppose further that

(1) the right adjoint $G: \mathcal{D} \rightarrow \mathcal{C}$ commutes with sequential colimits; and

(2) every cofibration in $\mathcal{D}$ with the LLP with respect to all fibrations is a weak equivalence.

Then $\mathcal{D}$ becomes a cofibrantly generated model category. Furthermore the sets $\{F i \mid i \in I\}$ and $\{F j \mid j \in J\}$ generate the cofibrations and the acyclic cofibrations of $\mathcal{D}$ respectively.

The proof, which makes repeated use of the small object argument, has been presented many places. See $[\mathbf{2 0}]$, Chapter II, for example. See also $[\mathbf{2 1}]$ and $[\mathbf{2 2}]$. But all these arguments are riffs of the argument in [30], §II.4.

EXAmple 3.7. Some hypothesis is needed, as it is not always possible to lift model category structures in this way. If the conclusion of Theorem 3.6 holds and the category $\mathcal{D}$ does have the indicated model category structure, then a simple adjointness argument shows that the left adjoint $F$ must preserve all weak equivalences between cofibrant objects. We give an example where this does not hold.

Let $\mathcal{C}=\mathbf{C h}_{*} k$ where $k$ is a field of characteristic 2 and let $\mathcal{D}=\mathbf{D G A} \mathbf{A}_{k}$ denote the category of non-negatively graded commutative differential graded algebras. Then the forgetful functor $\mathbf{D G} \mathbf{A}_{k} \rightarrow \mathbf{C h}_{*} k$ has a left adjoint $S$ given by the the symmetric algebra functor, with differential extended by the Leibniz rule. Every object in $\mathbf{C h}_{*} k$ is cofibrant. If $D(n) \in \mathbf{C} \mathbf{h}_{*}$ is the chain complex with one copy of $k$ in degrees $n$ and $n-1$ and identity boundary, then $0 \rightarrow D(n)$ is a weak equivalence. However, $S(0)=k \rightarrow S(D(n))$ is not a weak equivalence. Indeed if $y \in D(n)_{n}$ is non-zero, then $y^{2} \in S(D(n))$ is a cycle of degree $2 n$ which is not a boundary.

If, on the other hand, $k$ is a field of characteristic zero, then $\mathbf{D G} \mathbf{A}_{k}$ does inherit a model category structure in this way.

There is a useful criterion for checking hypothesis (2) of Theorem 3.6, also due to Quillen in his original work [30] and recently highlighted in [2].

THEOREM 3.8. Suppose in the category $\mathcal{D}$ (which is not yet a model category) the following two conditions hold:

(1) there is a functorial fibrant replacement functor; and

(2) every object has a natural path object. In otherwords we have a natural diagram for all $B \in \mathcal{D}$




where $i$ is a weak equivalence and $q$ is a fibration.

Then every cofibration in $\mathcal{D}$ with the LLP with respect to all fibrations is a weak equivalence.

There is a standard situation where path objects automatically exist - namely, when $\mathcal{D}$ is a simplicial category. See Corollary 4.14 .

\section{Simplicial Algebras and Resolutions in Non-abelian Settings}

4.1. The Dold-Kan theorem and simplicial resolutions. In Example 3.7 , we showed that the free commutative $R$-algebra functor, extending to chain complexes, does not generally preserve weak equivalences. As a consequence, differential graded algebras do not provide a good setting for creating resolutions of commutative algebras - except in characteristic zero. However, quite early on, Dold $[\mathbf{9}]$ noticed that the free commutative algebra functor $d i d$ preserve weak equivalences between cofibrant simplicial $R$-modules and that, therefore, simplicial algebras can provide a suitable setting for resolutions. This was extended and discussed further by Dold and Puppe in [10], which, in effect, talked about total derived functors before Quillen put a name to that concept.

In this section, we will begin with a discussion of simplicial $R$-modules in order to set the stage for discussing other sorts of categories of simplicial objects. The observation is that the category of simplicial $R$-modules is equivalent to the category of non-negatively graded chain complexes over $R$, so that one can work equally well with either category. Once this discussion is in place, we'll define a notion of resolution of a commutative algebra.

Let $s \operatorname{Mod}_{R}$ be the category of simplicial $R$-modules and $X \in s \operatorname{Mod}_{R}$. Then we defined the normalized chain complex of $X$ by eliminating the degenerate simplices as follows:

and setting

$$
N X_{n}=\frac{X_{n}}{s_{0} X_{n-1}+\cdots+s_{n-1} X_{n-1}}
$$

$$
\partial=\sum_{0}^{n}(-1)^{n} d_{i}: N X_{n} \longrightarrow N X_{n-1} .
$$

It is an exercise to show that the homomorphism $\partial$ is well defined and that

$$
H_{*} N X \cong H_{*}\left(X, \sum_{0}^{n}(-1)^{n} d_{i}\right) .
$$

There is an isomorphic formulation of $N X$. (See Example 2.15.) The composition

$$
N^{\prime} X_{n} \stackrel{\text { def }}{=} \bigcap_{i=1}^{n} \operatorname{Ker}\left\{d_{i}: X_{n} \longrightarrow X_{n-1}\right\} \stackrel{\subseteq}{\longrightarrow} X_{n} \longrightarrow N X_{n}
$$

is an isomorphism of $R$-modules and the homomorphism $d_{0}: N^{\prime} X_{n} \rightarrow N^{\prime} X_{n-1}$ yields an isomorphism of chain complexes. We immediately drop the distinction between $N X$ and $N^{\prime} X$ and we note that Example 2.15 implies that $H_{*} N X$ is naturally isomorphic to the homotopy groups of the geometric realization of $X$; hence we write $\pi_{*} X=H_{*} N X$.

THEOREM 4.1 (Dold-Kan). The normalized chain complex functor

$$
N: \operatorname{sMod}_{R} \longrightarrow C h_{*} R
$$


is an equivalence of categories.

The inverse functor to $N$ is easy to write down. Define $K: \mathbf{C h}_{*} R \rightarrow s \operatorname{Mod}_{R}$ by the formula

$$
\left(K C_{\bullet}\right)_{n}=\bigoplus_{\phi:[n] \rightarrow[m]} \phi^{*} C_{m}
$$

where the $\phi$ are surjections in $\boldsymbol{\Delta}$. For example, $\left(K C_{\bullet}\right)_{0}=C_{0},\left(K C_{\bullet}\right)_{1}=C_{1} \oplus s_{0} C_{0}$, and

$$
\left(K C_{\bullet}\right)_{2}=C_{2} \oplus s_{0} C_{1} \oplus s_{1} C_{1} \oplus s_{0} s_{0} C_{0} .
$$

The action of the degeneracy maps on $K C$ • is determined by these formulas and the simplicial identities. To get the action of the face maps $d_{i}, i>0$, we use the simplicial identities and require that $d_{i}=0$ on $C_{n} \subseteq\left(K C_{\bullet}\right)_{n}$. Finally to get the action of $d_{0}$, we use the simplicial identities and require that $d_{0}=\partial$ on $C_{n} \subseteq\left(K C_{\bullet}\right)_{n}$.

The Dold-Kan Theorem and Theorem 1.5 gives a model category structure on $s \operatorname{Mod}_{R}$ by transport of structure. If $X$ is a simplicial object in a category $\mathcal{C}$, the underlying degeneracy diagram neglects the face maps. More formally, if $\Delta_{+}$is the subcategory of the ordinal number category $\Delta$ with the same objects, but only surjective morphisms, then the underlying degeneracy diagram of $X: \Delta^{o p} \rightarrow \mathcal{C}$ is the restriction of $X$ to $\Delta_{+}^{o p}$.

Proposition 4.2. The category $s \operatorname{Mod}_{R}$ has the structure of a model category where a morphism $f: X \rightarrow Y$ is

(1) a weak equivalence if $\pi_{*} X \rightarrow \pi_{*} Y$ is an isomorphism;

(2) a fibration if $N X_{n} \longrightarrow N Y_{n}$ is onto for $n \geq 1$; and

(3) a cofibration if the underlying morphism of degeneracy diagrams is isomorphic to a morphism of the form

$$
X_{n} \longrightarrow Y_{n}=X_{n} \oplus \bigoplus_{\phi:[n] \rightarrow[k]} \phi^{*} P_{k}
$$

where all $P_{k}$ are projective.

It is possible to give an intrinsic description of the fibrations in $s \mathbf{M o d}_{R}$; that is, a description that does not appeal to the normalization functor. See Proposition 1.23 .

Lemma 4.3. Let $f: X \rightarrow Y$ be a morphism in the category $s \mathbf{M o d}_{R}$ of simplicial $R$-modules. Then the following are equivalent.

(1) The morphism $f$ is a fibration in $s \mathbf{M o d}_{R}$.

(2) The morphism $f$ is a fibration of simplicial sets.

(3) The induced map $X \rightarrow \pi_{0} X \times_{\pi_{0} Y} Y$ is a surjection.

We next turn to the question of what it means for an $R$-module $M$ to have a resolution in simplicial $R$-modules. If $X$ is a simplicial object in any category, an augmentation is a morphism $d_{0}: X_{0} \rightarrow A$ so that

$$
d_{0} d_{0}=d_{0} d_{1}: X_{1} \longrightarrow A \text {. }
$$

In the case of simplicial modules, we automatically obtain a $R$-module map $\pi_{0} X \rightarrow$ $A$. 
Definition 4.4. Let $M \in \operatorname{Mod}_{R}$. Then a simplicial resolution for $M$ is an augmented simplicial $R$-module $X \rightarrow M$ such that

(1) $X_{n}$ is a projective $R$-module for all $n \geq 0$; and

(2) the augmentation induces an isomorphism of $R$-modules

$$
\pi_{k} X \cong\left\{\begin{array}{cc}
M & k=0 \\
0 & k \neq 0
\end{array}\right.
$$

Equivalently, a simplicial resolution for $M$ is a cofibrant replacement for $M$ in $s \operatorname{Mod}_{R}$.

Now Theorem 4.1 immediately yields the following observation. Note that if $X \rightarrow M$ is an augmented simplicial $R$-module, then $N X \rightarrow M$ is an augmented chain complex.

Lemma 4.5. Let $M$ be an $R$-module. Then $X \rightarrow M$ is a simplicial resolution of $M$ if and only if $N X \rightarrow M$ is a projective resolution of $M$.

Now we turn to resolutions in a more general setting. At the beginning of section 1, we developed the notion of a resolution of an $R$-module by specifying a class of projectives or, equivalently, a class of surjections. Then a resolution was an acyclic chain complex of projectives; such were built by using the fact there were enough projectives.

Now let $\mathbf{A} \lg _{R}$ be the category of commutative $R$-algebras. An obvious class of projective objects in $\mathbf{A l g}_{R}$ are the free commutative algebras on projective modules $P$; that is, algebras of the form $S_{R}(P)$ where $S_{R}$ is the symmetric $R$-algebra functor. ${ }^{4}$ Then we should take the surjections in $\mathbf{A l g}_{R}$ to be the onto maps. Resolutions will be augmented simplicial objects in $s \mathbf{A l g}_{R}$; the following example implies that any simplicial $R$-algebra $T$ has a natural augmentation, in $R$-algebras, to $\pi_{0} Y$.

EXAmple 4.6. If $Y$ is a simplical $R$-algebra, then

$$
\pi_{0} Y=Y_{0} /\left(\operatorname{Im}\left(d_{0}-d_{1}\right)\right) .
$$

But, because of the presence of the degeneracy morphism $s_{0}$, the $R$-module $\operatorname{Im}\left(d_{0}-\right.$ $\left.d_{1}\right)$ is an ideal in $Y_{0}$ : if $a \in Y_{0}$ and $x=\left(d_{0}-d_{1}\right)(y)$, then

$$
\begin{aligned}
\left(d_{0}-d_{1}\right)\left(s_{0}(a) y\right) & =d_{0} s_{0}(a) \cdot d_{0}(y)-d_{1} s_{0}(a) d_{1}(y) \\
& =a\left(d_{0}(y)-d_{1}(y)\right) \\
& =a\left[\left(d_{0}-d_{1}\right)(y)\right]
\end{aligned}
$$

since the $d_{i}$ are algebra morphisms and $d_{i+1} s_{i}=d_{i} s_{i}=1$.

Next, a bit of notation. Let $X$ be a simplicial object in any category. We will write $X_{+}$for the underlying degeneracy diagram; that is, if $\Delta_{+}$is the subcategory of the ordinal number category where the morphisms are the surjections, then $X_{+}$ is $X$ restricted to $\Delta_{+}^{o p}$.

Definition 4.7. A resolution of $A \in \mathbf{A l g}_{R}$ is an augmented simplicial $R$ algebra $X \rightarrow A$ so that:

(1) there is a functor $P: \Delta_{+}^{o p} \rightarrow \operatorname{Mod}_{R}$ so that $P_{n}$ is projective for all $n$ and so that there is an isomorphism of diagrams of $R$-algebras $X_{+} \cong S_{R}(P)$

${ }^{4}$ If $P=R^{n}$ with basis $\left\{x_{i}\right\}$, then $S_{R}(P) \cong R\left[x_{1}, \ldots, x_{n}\right]$. 
(2) the augmentation induces an isomorphism

$$
\pi_{k} X \cong\left\{\begin{array}{cc}
A & k=0 \\
0 & k \neq 0
\end{array}\right.
$$

REMARK 4.8. 1.) In point (1) of this definition,we are requiring more than that $X$ be level-wise projective; we are requiring that the degeneracy maps respect this fact is a systematic way. This should be compared to Definition 4.4.1 where we do not seem to make any such requirement. However, because $R$-modules form an abelian category, the analogous requirement would be automatic.

2.) We have said nothing about the existence or uniqueness of such resolutions, but we will see that such a resolution turns out to be a cofibrant replacement for $A$ in an appropriate model category structure on simplicial algebras, so Corollary 2.12 applies. See Proposition 4.21.

EXAMPLE 4.9. We will see that the adjoint pair

$$
S_{R}: s \operatorname{Mod}_{R} \longleftrightarrow s \mathbf{A l g}_{R}: \mathcal{O}
$$

(where $\mathcal{O}$ is the forgetful functor) will be a Quillen functor. This implies that $S_{R}$ preserves weak equivalences between cofibrant objects, but in this example we will be much more concrete in hopes of further explaining why it is worthwhile to use simplicial objects. These observations are directly from Dold's paper [9].

In any category, an augmented simplicial object $X \rightarrow X_{-1}$ has an extra degeneracy if there are morphisms $s_{-1}: X_{n} \rightarrow X_{n+1} n \geq-1$ so that the formulas of Lemma 1.11 still hold. This implies that if $X$ is a simplicial $R$-module, then the normalized augmented chain complex $N X$ has a chain contraction onto $X_{-1}$ given by setting $T=s_{-1}: N X_{n} \rightarrow N X_{n+1}$. The Dold-Kan correspondence implies that $X$ has an extra degeneracy if and only if $N X$ has a chain contraction.

Now, if $X$ is a simplicial $R$-module with an extra degeneracy, then $S_{R}(X)$ has an extra degeneracy and, hence $\pi_{*} S_{R}(X) \cong S_{R}\left(X_{-1}\right)$. In particular, if $X$ is cofibrant and $\pi_{*} X=0$, then $\pi_{*} S_{R}(X) \cong R$ in degree 0 . By contrast, if $C_{\bullet}$ is a chain complex with a chain contraction, the associated differential graded algebra $S_{R}\left(C_{\bullet}\right)$ does not inherit a contraction. Again, see Example 3.7.

The notion of an extra degeneracy can be generalized to the notion of a simplicial homotopy (see [28]) and we are using the observation that the functor $S_{R}$ preserves simplicial homotopies.

4.2. Simplicial model categories. In the previous section we tried to suggest that simplicial resolutions were an appropriate and natural generalization of projective resolutions for $R$-modules. In turns out, in addition, that categories of simplicial objects support a great deal of structure - in particular, they are enriched over simplicial sets - and it is extremely useful to have an interplay between this structure and any model category structure that may be around. This leads to the notion of a simplicial model category.

If $\mathcal{C}$ is a category, let $s \mathcal{C}$ denote the simplicial objects in $\mathcal{C}$. We will assume that $\mathcal{C}$ has all limits and colimits.

We first note that $s \mathcal{C}$ has an action by simplicial sets. If $K \in s$ Sets and $X \in s \mathcal{C}$, define $K \otimes X \in s \mathcal{C}$ by

$$
(K \otimes X)_{n}=\coprod_{K_{n}} X_{n}
$$


The face and degeneracy maps are determined by those in $K$ and $X$. This construction gives a bifunctor $s$ Sets $\times s \mathcal{C} \longrightarrow s \mathcal{C}$ and one easily checks that there are natural isomorphisms

$$
(K \times L) \otimes X \cong K \otimes(L \otimes X)
$$

and

$$
\Delta^{0} \otimes X=* \otimes X \cong X .
$$

Less obvious, but equally formal is that there is also an exponential bifunctor

$$
\begin{aligned}
s \mathcal{C} \times s \operatorname{Sets}^{o p} & \longrightarrow \mathcal{C} \\
(Y, K) & \longmapsto Y^{K}
\end{aligned}
$$

determined by the adjoint formula

$$
s \mathcal{C}(K \otimes X, Y) \cong s \mathcal{C}\left(X, Y^{K}\right) .
$$

From this data we construct a simplicial mapping space. Let $X, Y \in s \mathcal{C}$, define $\operatorname{map}_{s \mathcal{C}}(X, Y)_{n} \in s$ Sets by

$$
\operatorname{map}_{s \mathcal{C}}(X, Y)_{n}=s \mathcal{C}\left(\Delta^{n} \otimes X, Y\right) .
$$

A morphism $\phi$ in the ordinal number category determines a morphism $\phi_{*}: \Delta^{m} \rightarrow$ $\Delta^{n}$ which, in turn, gives $\operatorname{map}_{s \mathcal{C}}(X, Y)$ its structure as a simplicial set. The formulas of Equations 4.3 and 4.4 supply an associative composition

$$
\operatorname{map}_{s \mathcal{C}}(Y, Z) \times \operatorname{map}_{s \mathcal{C}}(X, Y) \longrightarrow \operatorname{map}_{s \mathcal{C}}(X, Z) .
$$

and a natural isomorphism

$$
\operatorname{map}_{s \mathcal{C}}(X, Y)_{0}=s \mathcal{C}(X, Y) .
$$

Thus we have shown that $s \mathcal{C}$ is enriched over simplicial sets. But more is true: there are enriched adjoint isomorphisms

$$
\operatorname{map}_{s \mathcal{C}}(K \otimes X, Y) \cong \operatorname{map}_{\mathcal{S C}}\left(X, Y^{K}\right) \cong \operatorname{map}_{s \operatorname{Sets}}\left(K, \operatorname{map}_{s \mathcal{C}}(X, Y)\right) .
$$

All this data together gives $s \mathcal{C}$ the structure of a simplicial category, as defined by Quillen in [30] §II.2.

EXAMPLES 4.10 (Examples of simplicial categories). 1. If $s \mathcal{C}=s$ Sets, then $K \otimes X=K \times X$ and

$$
X^{K}=\operatorname{map}_{s \text { Sets }}(K, X) .
$$

This example predates Quillen, of course.

2. If $s \mathcal{C}=s \operatorname{Mod}_{R}$, then

$$
K \otimes X=R[K] \otimes_{R} X
$$

where $R[-]$ is the free $R$-module functor extended level-wise to simplicial sets. In addition

$$
X^{K}=\operatorname{map}_{s \operatorname{Sets}}(K, X)
$$

with addition and $R$-module action coming from the target. Similarly, if $X \in s \mathbf{A} \mathbf{l g}_{R}$ where $\mathbf{A l g}_{R}$ is the category of commutative $R$-algebras, then $X^{K}=\operatorname{map}_{s \operatorname{Sets}}(K, X)$ with algebra structure arising from the target. In this case $K \otimes X$ must be calculated using Equation 4.2. But this is not so bad, the coproduct in commutative $R$-algebras is given by tensor product.

3. This last example can be greatly expanded to almost any reasonable algebraic structure. Thus, to name just a few cases, there is a simplicial model category 
structure on simplicial associative algebras, simplicial Lie algebras, simplicial restricted Lie algebras over a field of positive characteristic, simplicial algebras over any operad in sets, and simplicial unstable algebras over the Steenrod algebra. All of these examples have appeared in the literature.

4. The category CGH of compactly generated weak Hausdorff spaces is a simplicial category. If $K$ is a simplicial set and $X \in \mathbf{C G H}$, set $K \otimes X=|K| \times X$ and $X^{K}=X^{|K|}$, where the $X^{|K|}$ denotes the exponential object in CGH. The reader sensitive to category theory will point out that $\mathbf{C G H}$ is really a topological category - defined by analogy with simplicial category - to which we respond that every topological category is a simplicial category via geometric realization.

5. Any simplicial category has a ready-made and natural candidate for a path object; namely, $X^{\Delta^{1}}$. Path objects arose in Theorem 3.8.

We would now like to meld the notion of simplicial structure with the structure of a model category when it is present. This is encoded in the following axiom, which in the literature is known as Quillen's SM7.

Definition 4.11 (Corner Axiom). Let $\mathcal{C}$ be a category which is at once a model category and a simplicial category. Then $\mathcal{C}$ is a simplicial model category if for $j: A \longrightarrow B$ a cofibration and $q: X \longrightarrow Y$ a fibration, the natural map of simplicial mapping spaces

$$
\operatorname{map}_{\mathcal{C}}(B, X) \longrightarrow \operatorname{map}_{\mathcal{C}}(B, Y) \times \operatorname{map}_{\mathcal{C}}(A, Y) \operatorname{map}_{\mathcal{C}}(A, X)
$$

is a fibration of simplicial sets which is a weak equivalence if $j$ or $q$ is a weak equivalence in $\mathcal{C}$.

The corner axiom can be reformulated in terms of the action of simplicial sets on $\mathcal{C}$ - often giving a condition which is easier to check. The proof is an easy exercise in adjointness arguments.

Proposition 4.12. Let $\mathcal{C}$ be a model category. The corner axiom is equivalent to both of the following two statements.

1. Let $j: A \rightarrow B$ be a cofibration in $\mathcal{C}$ and $i: K \rightarrow L$ be a cofibration of simplicial sets. Then

$$
i \otimes j: L \otimes A \sqcup_{K \otimes A} K \otimes B \longrightarrow L \otimes B
$$

is a cofibration in $\mathcal{C}$ which is a weak equivalence if $i$ or $j$ is.

2. Let $q: X \rightarrow Y$ be a fibration in $\mathcal{C}$ and $i: K \rightarrow L$ a cofibration of simplicial sets. Then

$$
X^{L} \longrightarrow X^{K} \times_{Y^{K}} Y^{L}
$$

is a fibration in $\mathcal{C}$ which is a weak equivalence if $q$ or $i$ is.

If $\mathcal{C}$ happens to be cofibrantly generated, there can be a further reduction in Proposition 4.12.1: we need only check the condition on $i \otimes j$ where $i$ and $j$ run over the generating cofibrations (or acyclic cofibrations, as needed) for $\mathcal{C}$ and for $s$ Sets. This is because, being a left adjoint in both variables $(-) \otimes(-)$ commutes with all colimits. These observations make it relatively easy to prove that there are examples of simplicial model categories:

Theorem 4.13. The categories $s$ Sets, $s \mathbf{M o d}_{R}$, and $\mathbf{C G H}$, with the simplicial structures of Example 4.10 become simplicial model categories. 
We close this section with two remarks meant to underscore the fact that simplicial model categories have the right sort of rich structure for us.

COROLlary 4.14. Let $\mathcal{C}$ be a simplicial model category.

(1) If $X \in \mathcal{C}$ is cofibrant, then

$$
X \amalg X \cong \Delta^{1} \otimes X \longrightarrow \partial \Delta^{1} \otimes X \longrightarrow * \otimes X \cong X
$$

is a natural cylinder object for $X$.

(2) If $X$ in $\mathcal{C}$ is fibrant, then

$$
X \longrightarrow X^{\Delta^{1}} \longrightarrow X^{\partial \Delta^{1}} \cong X \times X
$$

is a natural path object for $X$.

The next result says that the mapping space is a rich homotopical object. Indeed, one of the lessons of the last thirty years or so is that in order to compute homotopy classes of maps, the best strategy can be to compute the homotopy type of the mapping space, then read off the components.

Corollary 4.15. Let $\mathcal{C}$ be a simplicial model category. If $X \in \mathcal{C}$ is cofibrant and $Y \in s \mathcal{C}$ is fibrant, then

$$
\pi_{0} \operatorname{map}_{\mathcal{C}}(X, Y)=\mathbf{H o}(\mathcal{C})(X, Y)=[X, Y]_{\mathcal{C}} .
$$

REMARK 4.16. In a series of important papers, Dwyer and Kan noted that given any model category $\mathcal{C}$, there is a mapping simplicial set $L_{H} \mathcal{C}(X, Y)$ between two objects $X$ and $Y$ of $\mathcal{C}$ with the properties that

(1) $\pi_{0} L_{H}(X, Y)=[X, Y]_{\mathcal{C}}$, and

(2) if $\mathcal{C}$ is a simplicial model category, $X$ is cofibrant, and $Y$ is fibrant, there is a natural zig-zag of weak equivalences from $\operatorname{map}_{\mathcal{C}}(X, Y)$ to $L_{H}(X, Y)$.

This is the Dwyer-Kan hammock localization of $\mathcal{C}$. They point out, in fact, that one really only needs the weak equivalences to define $L_{H}(X, Y)$. See $[\mathbf{1 1}],[\mathbf{1 2}]$, and [13]. Of course, the simplicial mapping $\operatorname{space}_{\operatorname{map}_{\mathcal{C}}}(X, Y)$ is more concrete and the corner axiom of Definition 4.11 and its adjunct Proposition 4.12 are very useful for computations.

4.3. Simplicial algebras. We'd now like to pull together the threads from cofibrantly generated model categories and simplicial model categories. Here is an example of the sort of result we'd like to prove. The model category on simplicial $R$-modules was discussed in Proposition 4.2. The following result leaves open a characterization of cofibrations. While they will be formally determined - see Remark 1.4.2 - we want to be more concrete. This will be addressed below.

THEOREM 4.17. Let $R$ be a commutative ring and $s \mathbf{A l g}_{R}$ the category of simplicial commutative algebras over $R$. Then $s \mathbf{A l g}_{R}$ has the structure of a simplicial model category where a morphism $f: X \rightarrow Y$ is

(1) a weak equivalence if $\pi_{*} X \rightarrow \pi_{*} Y$ is an isomorphism and

(2) a fibration if the induced map $X \rightarrow \pi_{0} X \times_{\pi_{0} Y} Y$ is a surjection.

Proof. We would like to apply Theorem 3.6 to the adjoint pair

$$
S_{R}: s \operatorname{Mod}_{k} \rightleftarrows s \operatorname{Alg}_{k}: \mathcal{O}
$$

where $\mathcal{O}$ is the forgetful functor and $S_{R}$ is the symmetric algebra functor. Note that, by Lemma 4.3, we have defined a morphism $f \in s \mathbf{A l g}_{R}$ to be a weak equivalence 
or fibration if and only if $\mathcal{O}(f)$ is a weak equivalence or fibration. Since $S(-)$ commutes with all filtered colimits, we can appeal to Theorem 3.8 to complete the model category structure. To check that result, we turn to Corollary 4.14. If $B \in s \mathbf{A} \lg _{R}$, then $B^{\Delta^{1}}$ is a path object for $B$ in $s \mathbf{M o d}_{R}-$ because $s \mathbf{M o d}_{R}$ is already a simplicial model category. It follows immediately that $B^{\Delta^{1}}$ is a path object in $s \mathbf{A} \mathbf{l g}_{R}$. Since every object of $s \mathbf{A} \mathbf{l g}_{R}$ is fibrant, Theorem 3.8 applies, and we have our model category structure.

To a get a simplicial model category structure, we appeal to Proposition 4.12.2. Again we note that it is sufficient to check the condition there in $s \mathbf{M o d}_{R}$.

REMARK 4.18. The reader will have remarked that the previous argument is very formal and has wide application. In particular, we have simplicial model category structures on simplicial associative algebras, simplicial Lie algebras, simplicial groups, and so on.

The next point is to characterize cofibrations in $s \mathbf{A l g}_{R}$. Again, Theorem 3.6 can help, and this gives a chance to introduce a new construction and some other new ideas.

Let $I$ be a small category, $\mathcal{C}$ any category with colimits and $\mathcal{C}^{I}$ the category of $I$-diagrams in $\mathcal{C}$. Let $I^{\delta}$ be the category with same objects as $I$ but only identity morphisms; thus $I^{\delta}$ is $I$ made discrete. An $I$-diagram $X: I \rightarrow \mathcal{C}$ is $I$-free (or simply free) if it is the left Kan extension of some diagram $Z: I^{\delta} \rightarrow \mathcal{C}$. In formulas, this means that

$$
X_{i}=\underset{j \rightarrow i}{\sqcup} Z_{j}
$$

with the coproduct over all morphisms $j \rightarrow i$ in $I$.

DeFinition 4.19 . Let $\Delta$ be the ordinal number category and $\Delta_{+} \subset \Delta$ the category with same objects but only surjective morphisms. Let $\mathcal{C}$ be a category and $X: \Delta^{o p} \rightarrow \mathcal{C}$ a simplicial object. Then $X$ is $s$-free if the underlying diagram

$$
X_{+}: \Delta_{+}^{o p} \longrightarrow \mathcal{C}
$$

is free. More generally, a morphism $X \rightarrow Y$ is of simplicial objects is $s$-free if the underlying morphism $X_{+} \rightarrow Y_{+}$of $\Delta_{+}^{o p}$ diagrams is isomorphic to the inclusion of a summand $X_{+} \rightarrow X_{+} \amalg Y_{0}$ where $Y_{0}$ is $s$-free.

To rephrase, to say that $X$ is $s$-free is to say that there are objects $Z_{k}$ so that there are isomorphisms

$$
X_{n}=\coprod_{\phi:[n] \rightarrow[k]} \phi^{*} Z_{k}
$$

where $\phi$ runs over the surjections in $\Delta$. In this case we say $X$ is $s$-free on $\left\{Z_{k}\right\}$. More generally, a morphism can be $s$-free on a set of objects.

Definition 4.20. A morphism $X \rightarrow Y$ of simplicial $R$-algebras is free ${ }^{5}$ if it is $s$-free on a set of objects $\left\{S_{R}\left(P_{k}\right)\right\}$ where each $P_{k}$ is a projective $R$-module.

The following result characterizes cofibrations in $s \mathbf{A l g}_{R}$. It also shows that if we regard an $R$-algebra $A$ as a constant simplicial $R$-algebra, then it has a resolution in the sense of Definition 4.7.

\footnotetext{
${ }^{5}$ The terminology, while not fabulous, is in [30] and, thus, hallowed by history.
} 
Proposition 4.21. A morphism $X \rightarrow Y$ in $s \mathbf{A l g}_{R}$ is a cofibration if and only if it is a retract of a free morphism. Furthermore, any morphism can be factored naturally as

$$
X \stackrel{i}{\longrightarrow} Z \stackrel{p}{\longrightarrow} Y
$$

where $i$ is a free map and $p$ is an acyclic fibration. A similar statement applies to the "acyclic cofibration-fibration" factorization as well.

Proof. Note that free maps are closed under coproducts, cobase change, and sequential colimits. Since the generating cofibrations and the generating acyclic cofibrations are free maps, the statement about the factorizations follows from the small objects argument. Then if $f: A \rightarrow B$ is any cofibration, we obtain a diagram and a lifting problem



where $i$ is a free map and $p$ is an acyclic fibration. Since $f$ is a cofibration, the lifting problem can be solved and we have that $f$ is a retract of $i$. Hence every cofibration is a retract of a free map. It remains to be shown that every free map is a cofibration. This will be done below, when we have the skeletal decomposition. See Lemma 5.4.

4.4. Homology and Cohomology. To illustrate the efficacy of model categories, we will give Quillen's definition of the homology of commutative algebras. The paper [29] is still a wonderful read. This was one of the first real applications of model categories, and the proof of the transitivity sequence (Proposition 4.32) is most easily given using all the language of the theory.

Definition 4.22. If $\mathcal{C}$ is a category, $A \in \mathcal{C}$ is an abelian object if $\mathcal{C}(-, A)$ is naturally an abelian group. Assuming $\mathcal{C}$ has enough limits, this is equivalent to there being a multiplication morphism

$$
m: A \times A \longrightarrow A
$$

an identity $\epsilon: * \longrightarrow A$, where $*$ is the terminal object, and an inverse $i: A \longrightarrow A$ such that all the usual diagrams commute.

We will let $\mathcal{C}_{a b}$ denote the subcategory of abelian objects in $\mathcal{C}$.

EXAMPLES 4.23. 1.) If $\mathcal{C}$ is the category of sets, then $\mathcal{C}_{a b}$ is the category of abelian groups. If $\mathcal{C}=s$ Sets, then $\mathcal{C}_{a b}=s \mathbf{M o d}_{\mathbb{Z}}$.

2.) Fix a commutative ring $R$. The only abelian object in the category $\operatorname{Alg}_{R}$ of commutative $R$-algebras is the terminal obect 0 . To get more interesting examples we work over a fixed $R$-algebra $A$. Then if $M$ is an $A$-module, define a new $R$ algebra over $A$ by setting $A \ltimes M=A \oplus M$ with the multiplication

$$
(a, x)(b, y)=(a b, a y+b x) .
$$

Then

$$
\begin{aligned}
\left(\operatorname{Alg}_{R} / A\right)(X, A \ltimes M) & \cong \operatorname{Der}_{R}(X, M) \\
f=\epsilon \oplus \partial & \longmapsto \partial .
\end{aligned}
$$


Here $\operatorname{Der}_{R}(X, M)$ is the $R$-module of derivations; that is, of $R$-module maps $X \rightarrow$ $M$ satisfying the Leibniz rule. This equation displays $A \ltimes M$ as an abelian object. In fact, a simple exercise, shows that every abelian object in $\mathbf{A l g}_{R} / A$ is of this form. In particular, the functor $A \ltimes(-)$ defines equivalences of categories

$$
\operatorname{Mod}_{A} \rightarrow\left(\mathbf{A l g}_{k} / A\right)_{a b}
$$

and

$$
s \operatorname{Mod}_{A} \longrightarrow\left(s \mathbf{A l g}_{k} / A\right)_{a b} .
$$

For the next definition, we will suppose there are model category structures on $\mathcal{C}$ and $\mathcal{C}_{a b}$ so that the inclusion $\mathcal{C}_{a b} \longrightarrow \mathcal{C}$ is the right adjoint of a Quillen functor; that is, there is an abelianization functor

$$
\mathrm{Ab}: \mathcal{C} \longrightarrow \mathcal{C}_{a b}
$$

left adjoint to inclusion which preserves cofibrations and weak equivalences between cofibrant objects.

DEFinition 4.24. Homology is the total left derived functor of abelianization. That is, if $X \in \mathcal{C}$, then the Quillen homology of $X$ is the object $L \mathbf{A b}(X) \in \mathcal{C}_{a b}$.

EXAMPLE 4.25 (Homology of spaces). If $X \in s$ Sets, then $X$ is cofibrant and $L \mathbf{A b}(X)=\mathbb{Z} X$, the simplicial abelian group generated by $X$. In particular, we have that if $Y$ is a topological space

$$
\pi_{n} L \mathbf{A b}(S(Y))=\pi_{n} \mathbb{Z} S(Y) \cong H_{n}(Y ; \mathbb{Z})
$$

and we recover singular homology.

If $X$ is a topological space, then a cofibrant replacement for $X$ can be taken to be a weak equivalence $Y \rightarrow X$ with $Y$ cofibrant. Then $\mathbf{A b}(Y)$ is the free topological abelian group on $Y$ and the Dold-Thom Theorem implies that there are natural isomorphisms

$$
\pi_{*} L \mathbf{A} \mathbf{b}(X) \cong \pi_{*} \mathbf{A} \mathbf{b}(Y) \cong H_{*}(Y) \cong H_{*}(X) .
$$

EXAMPLE 4.26 (Homology of groups). If $G$ is a group, we may regard $G$ as a constant simplicial group, and then $L \mathbf{A b}(G)=\mathbf{A b}(X)=X /[X, X]$ where $X \rightarrow G$ is a cofibrant model for $G$ and $[X, X]$ is the commutator subgroup. On the other hand the homology of $G$ is usually defined to be

$$
\operatorname{Tor}_{*}^{\mathbb{Z}[G]}(\mathbb{Z}, \mathbb{Z})=H_{*}(B G)
$$

where $B G$ is the classifying space of $G$. The claim is that there is a degree-shifting isomorphism between $\pi_{*} L \mathbf{A b}(G)$ and $\tilde{H}_{*}(B G)$. To see this, form the bisimplicial abelian group $\mathbb{Z}[B X]$. This has two filtrations, and hence, two spectral sequences converging to the same graded abelian group.

For all $q, X_{q}$ is a free group. Thus, if we filter by the simplicial degree coming from $X$ we get a spectral sequence with $E^{1}$-term

$$
E_{p, q}^{1} \cong H_{q}\left(B X_{p}\right) \cong \begin{cases}X_{p} /\left[X_{p}, X_{p}\right] & q=1 \\ \mathbb{Z} & q=0 \\ 0 & q>1\end{cases}
$$

Thus $E_{p, 1}^{2}=\pi_{p} L \mathbf{A b}(G), E_{0,0}^{2}=\mathbb{Z}$ and $E_{p, q}^{2}=0$ for all other $p$ and $q$. One the other hand, if we filter by the simplicial degree coming from the functor $B(-)$ we 
get a spectral sequence with

$$
E_{p, q}^{1} \cong \begin{cases}\mathbb{Z}[B G] & p=0 \\ 0 & p \neq 0\end{cases}
$$

Thus $E_{0, q}^{2}=H_{q} B G$ and $E_{p, q}^{2}=0$ if $p \neq 0$. Thus we conclude that

$$
\pi_{n} L \mathbf{A} \mathbf{b}(G) \cong H_{n+1} B G \text {. }
$$

We now turn to the case of commutative algebras over a commutative ring $R$. The resulting homology theory is André-Quillen homology and it is the subject of a very extensive discussion elsewhere in these nots by Srikanth Iyengar [24], which the reader should turn to. We only include it here because (a) the construction of a suitable homology theory for commutative algerbas was one of the early successes of the theory of model categories and (b) the first author of this monograph is very fond of it.

The first question is what the abelianization functor for commutative algebras should be.

If $X$ is an $R$-algebra, let $I=\operatorname{Ker}\left(X \otimes_{R} X \rightarrow X\right)$ be the kernel of $R$-algebra multiplication and let

$$
\Omega_{X / R}=I / I^{2} \text {. }
$$

Then the morphism $d: X \rightarrow \Omega_{X / R}$ sending $y$ to the coset of $y \otimes 1-1 \otimes y$ is a derivation and the natural map

$$
\begin{aligned}
\operatorname{Mod}_{X}\left(\Omega_{X / R}, M\right) & \longrightarrow \operatorname{Der}_{R}(X, M) \\
f & \mapsto f \circ d
\end{aligned}
$$

is an isomorphism. Since $\Omega_{X / R}$ represents derivations, it is called the module of differentials.

Let $A$ be an $R$-algebra and $\mathbf{A} \lg _{R} / A$ the category of algebras over $A$. Recall that an object in $\mathbf{A l g}_{R} / A$ is a morphism of commutative $R$-algebras $B \rightarrow A$. In Example 4.23.2 we indicated that the category of abelian objects in $\mathbf{A l g}_{R} / A$ was equivalent to the category $\mathbf{M o d}_{A}$ and that the inclusion functor of abelian objects into $\mathbf{A l g}_{R} / A$ was naturally isomorphic to the functor

$$
A \ltimes(-): \operatorname{Mod}_{R} \longrightarrow \mathbf{A l g}_{R} / A .
$$

But there are natural isomorphisms

$$
\left(\operatorname{Alg}_{R} / A\right)(X, A \ltimes M) \cong \operatorname{Der}_{R}(X, M) \cong \operatorname{Mod}_{A}\left(A \otimes_{X} \Omega_{X / R}, M\right) .
$$

Therefore, we have proved the following result, which displays the differentials as the abelianization functor for $\mathbf{A l g}_{R} / A$.

Proposition 4.27. The functor

$$
X \mapsto A \otimes_{X} \Omega_{X / R}
$$

is left adjoint to the functor $M \mapsto A \ltimes M$ from $\operatorname{Mod}{ }_{A}$ to $\mathbf{A l g}_{R} / A$.

Note that the functor $M \mapsto A \ltimes M$ extends level-wise to a functor $s \mathbf{M o d}_{A} \rightarrow$ $s \mathbf{A l g}_{R} / A$. This functor preserves all weak equivalences and fibrations, so the adjoint

$$
\Omega_{(-) / R}: s \mathbf{A l g}_{R} / A \longrightarrow s \operatorname{Mod}_{A}
$$

preserves cofibrations and weak equivalences between cofibrant objects; that is, we have a Quillen functor. 
Definition 4.28. Let $A$ be a commutative $R$-algebra. Then the cotangent complex for $A$ is the simplicial $A$-module given by the total left derived functor of differentials:

$$
L_{A / R}=A \otimes_{X} \Omega_{X / R}
$$

where $X \rightarrow A$ is a cofibrant replacement for $A$ in the category $s \mathbf{A} \lg _{R} / A$. (Cofibrant objects in $s \mathbf{A} \lg _{R} / A$ can be understood using Proposition 4.21 and Example 1.7.) The André-Quillen homology of $A$ is given by

$$
D_{q}(A / R)=\pi_{q} L_{A / R}=H_{q} N L_{A / R} .
$$

More generally, if $M$ is an $A$-module, we set $D_{*}(A / R ; M)=\pi_{*}\left(M \otimes_{A} L_{A / R}\right)$.

REMARK 4.29. We write down here that there is a natural augmentation $L_{A / R} \rightarrow \Omega_{A / R}$

It may not be immediately obvious what the naturality properties of $L_{A / R}$ should be. The important observation is that the cotangent complex is a relative homology object; that is, a functor of the arrow $R \rightarrow A-$ or, we might say, of the pair $(A, R)$. If we have a commutative diagram

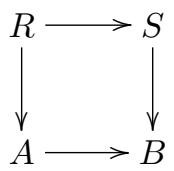

then we get a morphism $B \otimes_{A} L_{A / R} \rightarrow L_{B / S}$. To see this, form the following diagram, which can be chosen naturally:

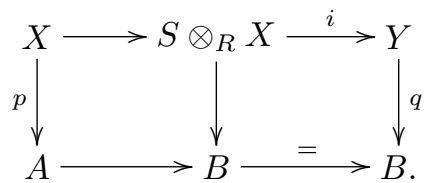

Here $p$ is an acyclic fibration in $R$-algebras with $X$ cofibrant, $i$ is a cofibration in $S$ algebras and $q$ is an acyclic fibration in $S$-algebras. Then $S \otimes_{R} X$ is a cofibrant $S$ algebra and, hence, $Y$ is a cofibrant $S$-algebra. Thus we may model $B \otimes_{A} L_{A / R} \rightarrow$ $L_{B / S}$ by

$$
B \otimes_{A}\left(A \otimes_{X} \Omega_{X / R}\right) \rightarrow B \otimes_{Y} \Omega_{Y / S}
$$

We might ask to what extent this sort of homology actually acts like homology. We'll give two answers. First, the next result gives some basic properties of this theory, including a result that says André-Quillen homology takes finite coproducts to sums. (The finite coproduct of commutative algebras is their tensor product.) Second, we'll also give below, in Theorem 4.32 a proof of the transitivity sequence, which is an analog of the long exact sequence of a pair.

LEMma 4.30. The cotangent complex has the following properties:

(1) if $A=S_{R}(P)$ where $P$ is a projective $R$-module than the natural augmentation $L_{A / R} \rightarrow \Omega_{A / R}$ is a weak equivalence of simplicial $A$-modules;

(2) if $A$ and $B$ are two $R$-algebras, let $C=A \otimes_{R} B$. If one of $A$ or $B$ is flat over $R$, then the natural map

$$
C \otimes_{A} L_{A / R} \oplus C \otimes_{B} L_{B / R} \longrightarrow L_{C / R}
$$

is an isomorphism. 
Proof. The proofs are easy and we include them only to illustrate the flexibility of model categories. The first statement follows from the fact that $A=S_{R}(P)$ is already cofibrant in $\operatorname{Alg}_{R} / A$. For the second statement, let $X \rightarrow A$ and $Y \rightarrow B$ be cofibrant replacements for $A$ and $B$ respectively. Then $X \otimes_{R} Y \rightarrow A \otimes_{R} B=C$ is a cofibrant replacement for $C$. To see this, note that (1) the coproduct of cofibrant objects is cofibrant and that (2) since $X$ is level-wise projective as an $R$-module, that flatness hypothesis implies

$$
\pi_{*}\left(X \otimes_{R} Y\right)=\pi_{*} X \otimes_{R} \pi_{*} Y \cong A \otimes_{R} B .
$$

The result now follows from the formula, easily checked, that

$$
\Omega_{\left(X \otimes_{R} Y\right) / R}=\left(X \otimes_{R} Y\right) \otimes_{X} \Omega_{X / R} \oplus\left(X \otimes_{R} Y\right) \otimes_{Y} \Omega_{Y / R} .
$$

A fundamental result about André-Quillen homology is the following, which is also easy to prove using model categories:

THEOREM 4.31 (Flat base change). Suppose there is a commutative diagram



with one of $i$ or $f$ flat. Let $B=S \otimes_{R} A$. Then the induced morphism of simplicial $B$-modules $B \otimes_{A} L_{A / R} \rightarrow L_{B / S}$ is a weak equivalence.

Proof. Choose a cofibrant model $X \rightarrow A$ for $A$ as an $R$-algebra. Then $S \otimes_{R}$ $X \rightarrow B$ is a weak equivalence, by the flatness assumption, and $S \otimes_{R} X$ is cofibrant. The result follows.

To set the stage for the next result, we consider a sequence of morphisms

$$
R \longrightarrow A \stackrel{f}{\longrightarrow} B
$$

of commutative rings. Then a standard result (and an easy exercise) about differentials is that there is an exact sequence

$$
B \otimes_{A} \Omega_{A / R} \longrightarrow \Omega_{B / R} \longrightarrow \Omega_{B / A} \rightarrow 0
$$

and that, furthermore, this sequence becomes short exact if $f: A \rightarrow B$ has an $R$-algebra retraction; that is, if there is an $R$-algebra map $r: B \rightarrow A$ so that $r f=1: A \rightarrow A$. In trying to develop a homology theory of commutative algebras, the question was how to extend this exact sequence to the left. To state a result, call a sequence of simplicial $R$-modules $M \rightarrow N \rightarrow P$ a cofiber sequence, if the composite is null-homotopic and if the mapping cone of the first map is weakly equivalent to $P$. Such a sequence induces a long exact sequence in homotopy.

Proposition 4.32. Let $R \rightarrow A \rightarrow B$ be a sequence of commutative rings. Then the composition

$$
B \otimes_{A} L_{A / R} \longrightarrow L_{B / R} \longrightarrow L_{B / A}
$$


is a cofiber sequence of simplicial $B$-modules. In particular, if $M$ is a $B$-module there is a long exact sequence

$$
\begin{aligned}
\cdots \rightarrow D_{1}(A / R ; M) & \rightarrow D_{1}(B / R ; M) \rightarrow D_{1}(B / A ; M) \\
& \rightarrow M \otimes_{A} \Omega_{A / R} \rightarrow M \otimes_{B} \Omega_{B / R} \rightarrow M \otimes_{B} \Omega_{B / A} \rightarrow 0 .
\end{aligned}
$$

Proof. The long exact sequence follows from the cofiber sequence, as $L_{B / A}$ will be level-wise a projective $B$-module. To prove that we have a cofiber sequence, choose an acyclic fibration $X \rightarrow A$ where $X$ is free. Then factor the composition $X \rightarrow A \rightarrow B$ to obtain a commutative square

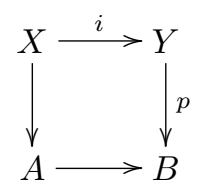

where $i$ is a free map and $p$ is an acyclic fibration. Then we have an exact sequence of simplicial $B$-modules

$$
0 \rightarrow B \otimes_{X} \Omega_{X / R} \rightarrow B \otimes_{Y} \Omega_{Y / R} \rightarrow B \otimes_{Y} \Omega_{Y / X} \rightarrow 0
$$

using that Equation 4.5 becomes short exact because $X_{n} \rightarrow Y_{n}$ has a retract for all $n$; indeed $X_{n} \rightarrow Y_{n}$ is isomorphic to

$$
X_{n} \longrightarrow X_{n} \otimes_{R} S_{R}\left(P_{n}\right)
$$

where $P_{n}$ is a projective $R$-module. To finish the argument, note that we have a map $A \otimes_{X} Y \rightarrow B$. This is a weak equivalence and $A \otimes_{X} Y$ is a cofibrant simplicial $A$-algebra. Thus we need only note that Equation 4.6 implies that

$$
B \otimes_{Y} \Omega_{Y / X} \rightarrow B \otimes_{\left(A \otimes_{X} Y\right)} \Omega_{\left(A \otimes_{X} Y\right) / A}
$$

is an isomorphism.

REMARK 4.33. André-Quillen homology is a very sensitive invariant of morphims of commutative rings or, more generally, of schemes. (See [23] for the schemetheoretic generalizations.) For example, suppose $A$ is a finitely generated $R$-algebra. Then $R \rightarrow A$ is smooth if and only if $L_{A / R} \rightarrow \Omega_{A / R}$ is a weak equivalence and $\Omega_{A / R}$ is projective as an $A$-module. In addition, $R \rightarrow A$ is étale if and only if $L_{A / R}$ has vanishing homology.

REMARK 4.34. There is a companion André-Quillen cohomology theory. If $M$ is an $A$-module, let $K(M, n) \in s \operatorname{Mod}_{A}$ be the unique simplicial $A$-module so that

Then

$$
N K(M, t)_{n}=\left\{\begin{array}{cc}
M, & n=t \\
0, & n \neq t
\end{array}\right.
$$

$$
\pi_{n} K(M, t) \cong H_{n} N K(M, t)=\left\{\begin{array}{cc}
M, & n=t \\
0, & n \neq t
\end{array}\right.
$$

The $n$-th André-Quilen cohomology group of $A$ is defined to be homotopy classes of maps into the resulting Eilenberg-MacLane algebra:

$$
\begin{aligned}
D^{n}(A / R ; M) & =\mathbf{H o}\left(s \operatorname{Alg}_{R} / A\right)(A, A \ltimes K(M, n)) \\
& \cong H^{n} \operatorname{Mod}_{A}\left(N L_{A / R}, M\right) \\
& \cong H^{n} \operatorname{Der}_{R}(X, M)
\end{aligned}
$$


Here $X \rightarrow A$ is a cofibrant replacement for $A$ as $R$-algebra. In fact, we can define the André-Quillen cohomology space by the equation

$$
\mathcal{H}^{n}(A / R ; M)=\operatorname{map}_{s \operatorname{Alg}_{R} / A}(X, A \ltimes K(M, n))
$$

where $X \rightarrow A$ is a cofibrant replacement. Then

$$
D^{n}(A / R ; M)=\pi_{0} \mathcal{H}^{n}(A / R ; M) \cong \pi_{t} \mathcal{H}^{n+t}(A / R ; M) .
$$

REMARK 4.35. André-Quillen (co-)homology arises naturally in homotopy theoretic settings: see for example, [1], [4], [18], and [19]. For example, if $X$ and $Y$ are spaces and $\phi: X \rightarrow Y$ is a chosen basepoint for the space of maps map $(X, Y)$, then we leave it as an exercise to make sense of the statement that there is a Hurewicz map

$$
\pi_{t}(\operatorname{map}(X, Y) ; \phi) \longrightarrow \operatorname{Der}_{\mathcal{K}}\left(H^{*} Y, \Sigma^{t} H^{*} X\right) .
$$

Here $H^{*}(-)$ is homology with $\mathbb{F}_{p}$-coefficients, $\mathcal{K}$ is the category of unstable algebras over the Steenrod algebra, and $\Sigma^{t} H^{*} X$ is an $H^{*} Y$ module via $\phi^{*}$. This is the edge homomorphism of the Bousfield-Kan spectral sequence, and the $E_{2}$-term is given by the higher derived functors of Der - in short, by André-Quillen cohomology.

\section{Resolutions in Model Categories}

In this section we turn to the project of creating and manipulating simplicial resolutions in model categories. In previous sections we worked with resolutions of modules and resolutions of algebras, but now we'd like to turn to resolving objects which, at least for us, are only inherently defined up to weak equivalence - for example, we might want to resolve topological spaces by spheres. This leads to the resolution model categories of Dwyer, Kan, and Stover. We will explain the approach developed by Bousfield.

5.1. Skeletons and the skeletal decomposition. This preliminary section will be devoted to developing the canonical and natural filtration of a simplicial object by its skeleton. This will give us a chance to introduce some notation and prove, for example, that every free morphism of simplicial algebras is a cofibration.

The skeletons are defined by introducing some subcategories of the ordinal number category $\Delta$. Let $\Delta[n] \subset \Delta$ be the full-subcategory with objects $[k], k \leq n$. If $\mathcal{C}$ is a category, we let $s_{n} \mathcal{C}$ be the category of contravariant functors from $\Delta[n]$ to $\mathcal{C}$. There is then a restriction functor $r_{*}: s \mathcal{C} \rightarrow s_{n} \mathcal{C}$ from simplicial objects in $\mathcal{C}$. If the category $\mathcal{C}$ has enough limits and colimits - which we will assume - this functor has a left adjoint, given by left Kan extension

$$
r^{*}: s_{n} \mathcal{C} \longrightarrow s \mathcal{C} \text {. }
$$

If $X \in s \mathcal{C}$ is a simplicial object we define the $n$th skeleton of $X$ by the formula

$$
\mathrm{sk}_{n} X=r^{*} r_{*} X \text {. }
$$

By the construction of Kan extensions, we have that

$$
\left(\operatorname{sk}_{n} X\right)_{m}=\operatorname{colim}_{\phi:[m] \rightarrow[k]} \phi^{*} X_{k}
$$

where $\phi$ runs over all the morphisms in $\Delta$ with $k \leq n$. But, since every such morphism factors uniquely as a surjection followed by an injection, we may take the colimit over the surjections in $\Delta$ with $k \leq n$. In particular, $\left(\operatorname{sk}_{n} X\right)_{m}=X_{m}$ if $m \leq n$ 
and you should think of $\operatorname{sk}_{n} X$ as the sub-simplicial set of $X$ generated by the nondegenerate simplices in degrees $k \leq n$. There are natural maps $\operatorname{sk}_{n} X \rightarrow \operatorname{sk}_{n+1} X$ and $\operatorname{sk}_{n} X \rightarrow X$. Furthermore, the natural map

$$
\operatorname{colim}_{n} \operatorname{sk}_{n} X \longrightarrow X
$$

is an isomorphism.

More generally, if $X \rightarrow Y$ is a morphism of simplicial objects in $\mathcal{C}$, we define the relative $n$-skeleton $\operatorname{sk}_{n}^{X} Y$ by the pushout diagram

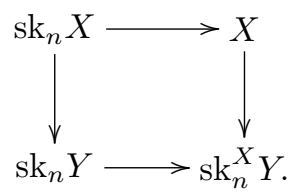

Then $\operatorname{sk}_{-1}^{X} Y=X$ and $\operatorname{colim}_{n} \operatorname{sk}_{n}^{X} Y=Y$.

While these definitions are formal enough, they have impact because we can explicitly describe the transition from the $(n-1)$ st skeleton to the $n$th skeleton. This is what we do next.

If $X$ is a simplicial set, we may define the degenerate $n$-simplices as the image of the degeneracies:

$$
\bigcup_{i} s_{i} X_{n-1} \subseteq X_{n}
$$

However, for more general simplicial objects we need a categorical definition of the degeneracies. As a hint, recall that the degeneracties in degree $n$ of a simplicial set can equally be written

$$
\bigcup_{\phi:[n] \rightarrow[k]} \phi^{*} X_{k}=\operatorname{colim}_{\phi:[n] \rightarrow[k]} X_{k}
$$

where $\phi$ runs over the non-indentity surjections in $\Delta$.

Definition 5.1. Let $\mathcal{C}$ be a category with all limits and colimits and let $X \in s \mathcal{C}$ be a simplicial object in $\mathcal{C}$. Then the $n$th latching object of $X$ is the colimit

$$
L_{n} X=\operatorname{colim}_{\phi:[n] \rightarrow[k]} X_{k}
$$

where $k$ runs over the non-identity surjections in the ordinal number category $\Delta$. The degeneracies define a natural map $s: L_{n} X \rightarrow X_{n}$.

There is also a companion matching object given by the limit

$$
M_{n} X=\lim _{\psi:[k] \rightarrow[n]} X_{k}
$$

where $\psi$ runs over the non-identity injections. The face maps yield a natural map $d: X_{n} \rightarrow M_{n} X$.

By comparing Equation 5.1 with the definition of the latching object we immediately have a natural isomorphism

$$
\left(\operatorname{sk}_{n-1} X\right)_{n}=L_{n} X .
$$

Now we give $s \mathcal{C}$ its standard simplicial structure, as in Equations 4.2 and following. A moment's thought should convince the reader that if $Z \in \mathcal{C}$ is regarded as a constant simplicial object then there are natural isomorphisms

$$
s \mathcal{C}\left(\Delta^{n} \otimes Z, Y\right) \cong \mathcal{C}\left(Z, Y_{n}\right)
$$


and

$$
s \mathcal{C}\left(\partial \Delta^{n} \otimes Z, Y\right) \cong \mathcal{C}\left(Z, M_{n} Y\right) .
$$

If we set $Y=\operatorname{sk}_{n} X$ and $Z=X_{n}$ in the first equation, we obtain a natural map

$$
\Delta^{n} \otimes X_{n} \longrightarrow \operatorname{sk}_{n} X
$$

and if we set $Y=\operatorname{sk}_{n-1} X$ and $Z=X_{n}$ in the second equation, we obtain a natural map

$$
\partial \Delta^{n} \otimes X_{n} \longrightarrow \operatorname{sk}_{n-1} X
$$

since $M_{n} \mathrm{sk}_{n-1} X=M_{n} X$. Setting $Z=L_{n} X$ and $Y=\mathrm{sk}_{n-1} X$ in the first equation we obtain a map

$$
\Delta^{n} \otimes L_{n} X \longrightarrow \operatorname{sk}_{n-1} X
$$

using Equation 5.2. In the end we obtain a commutative diagram

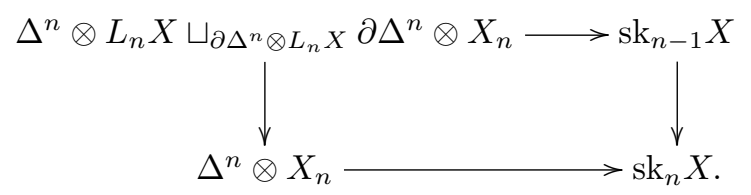

The claim, of course, is that this is a push-out. We can actually give a relative version as well. See [20] for an argument.

Proposition 5.2. Let $X \rightarrow Y$ be a morphism of simplicial objects and let

$$
L_{n}^{X} Y=X_{n} \sqcup_{L_{n} X} L_{n} Y .
$$

Then there is a push-out diagram

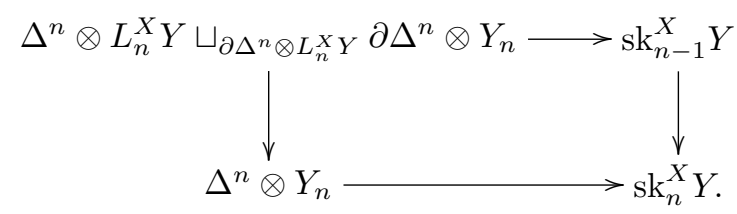

Proposition 5.2 has a considerable simplification if the morphism $X \rightarrow Y$ is $s$-free, as in Definition 4.19.

Proposition 5.3. Suppose a morphism $X \rightarrow Y$ in $s \mathcal{C}$ is s-free on a set of objects $Z_{k}, k \geq 0$. Then there is a push-out diagram

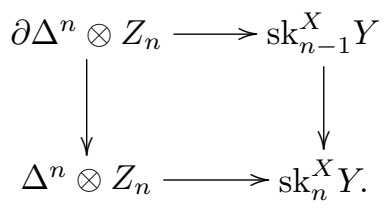

This follows from Proposition 5.2 and the following observation: if $X$ is $s$-free on $Z_{k}$, then

$$
L_{n} X \cong \underset{\phi:[n] \rightarrow[k]}{\amalg} \phi^{*} Z_{k}
$$

where $\phi$ runs over the non-identity surjections of $\Delta$. Compare Equation $4.1-$ this is the case of simplicial modules.

We can now prove the remaining implication of Proposition 4.21.

Lemma 5.4. Let $A \rightarrow B$ be a free map in the category $s \mathbf{A l g}_{R}$. Then $A \rightarrow B$ is a cofibration. 
Proof. We need to show that any lifting problem

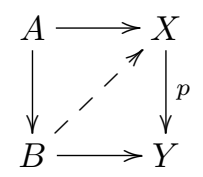

with $p$ an acyclic fibration of simplicial $R$-algebras has a solution. We induct over the relative skeletons of $A \rightarrow B$ and solve the lifting problems



By the definition of what it means to be free, the previous result provides a push-out square

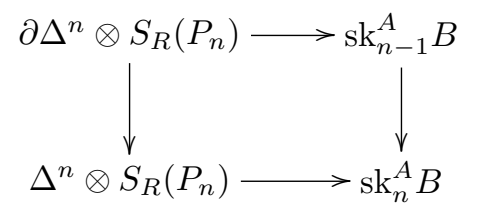

where $S_{R}(-)$ is the symmetric algebra functor and $P_{n}$ is a projective $R$-module. Now $K \otimes S_{R}\left(P_{n}\right)=S_{R}\left(K \otimes P_{n}\right)$, where $K \otimes(-)$ is interpreted in $R$-algebras or $R$-modules as required. Thus, we need only solve the lifting problems

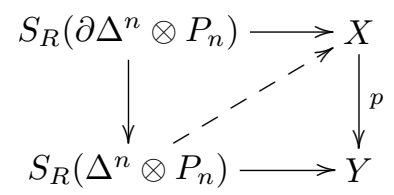

But this lifting problem in $s \mathbf{A} \mathbf{l g}_{R}$ is adjoint to the lifting problem

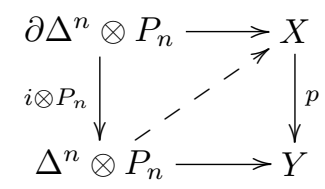

in $s \operatorname{Mod}_{R}$, and this problem has a solution since $p$ remains an acylic fibration in $s \operatorname{Mod}_{R}$ and $i \otimes P_{n}$ is a cofibration in $s \operatorname{Mod}_{R}$.

5.2. Reedy model categories; the invariance of realization. If $\mathcal{C}$ has enough colimits, then there is a notion of geometric realization from the category $s \mathcal{C}$ of simplicial objects in $\mathcal{C}$ back down to $\mathcal{C}$. In his thesis [32], Reedy noticed that if $\mathcal{C}$ is a model category, this could be made into a Quillen functor and, in particular, realization preserved weak equivalences between appropriately defined cofibrant objects. Since this is proved using the technology of the previous section, we'll give an exposition here. It also sets the stage for the resolution model categories of the next section.

THEOREM 5.5. Let $\mathcal{C}$ be a model category and let $s \mathcal{C}$ be the category of simplicial objects in $\mathcal{C}$. Then there is a model category structure on $s \mathcal{C}$ where a morphism $f: X \rightarrow Y$ is 
(1) a weak equivalence if $X_{n} \rightarrow Y_{n}$ is a weak equivalence in $\mathcal{C}$ for all $n \geq 0$;

(2) a cofibration if the natural morphism from the relative latching object

$$
L_{n}^{X} Y=X_{n} \sqcup_{L_{n} X} L_{n} Y \rightarrow Y_{n}
$$

is a cofibration in $\mathcal{C}$ for all $n \geq 0$; and

(3) a fibration if the natural morphism to the relative matching object

$$
X_{n} \rightarrow Y_{n} \times_{M_{n} Y} M_{n} X
$$

is a fibration in $\mathcal{C}$ for all $n \geq 0$.

The proof is in $[\mathbf{3 2}]$, but see also $[\mathbf{2 0}]$ and $[\mathbf{2 1}]$. We will refer to the weak equivalences, cofibrations, and fibrations in this model category structure as Reedy weak equivalences, etc.

REMARK 5.6. 1.) To get a feel for this model category structure, note that if $X \in \mathcal{C}$ is cofibrant, then the constant object on $X \in s \mathcal{C}$ is Reedy cofibrant. However, constant objects are hardly ever Reedy fibrant.

2.) This model category structure is not a simplicial model category structure in the standard simplicial structure on $s \mathcal{C}$. (See Equation 4.2 and following.) If $i: K \rightarrow L$ is a cofibration of simplicial sets and $j: X \rightarrow Y$ is a Reedy cofibration, then

$$
i \otimes j: K \otimes Y \sqcup_{K \otimes X} L \otimes X \rightarrow L \otimes Y
$$

is a Reedy cofibration which is a Reedy weak equivalence if $j$ is a Reedy weak equivalence; however, it need not be a weak equivalence if $i$ is a weak equivalence. Consider, for example, the inclusion

$$
d_{0} \otimes X: X=\Delta^{0} \otimes X \rightarrow \Delta^{1} \otimes X .
$$

However, if $\mathcal{C}$ is a simplicial model category structure, then $s \mathcal{C}$ inherits an "internal" simplicial model category structure promoted up from $\mathcal{C}$.

3.) One example we might take for $\mathcal{C}$ is the category $s$ Sets of simplicial sets. Then $s \mathcal{C}$ is the category of bisimplicial sets, but the Reedy model category structure priviledges the new simplicial direction. Note that every bisimplicial set is automatically Reedy cofibrant.

4.) At virtually the same time that Reedy was writing his thesis, Bousfield and Kan wrote their book [7]. Central to the existence of the homotopy spectral sequence of a cosimplicial space was a model category structure on cosimplicial simplicial sets. This is, it turns out, the Reedy structure, after you've taken the opposite category enough times. One of their theorems - the homotopy invariance of the total space of a fibrant cosimplicial space - is essentially Theorem 5.9 below.

5.) If $\mathcal{C}=\mathbf{C G H}$ is the category of compactly generated weak Hausdorff spaces, the notion of a Reedy cofibrant object is a variation on the notion of a proper simplicial space. For proper, one only requires that $L_{n} X \rightarrow X_{n}$ be a Hurewicz cofibration or, perhaps, only a closed inclusion.

Now suppose that $\mathcal{C}$ itself is a simplicial model category. Let us write

$$
(-) \otimes_{\mathcal{C}}(-): s \text { Sets } \times \mathcal{C} \rightarrow \mathcal{C}
$$

for the action of simplicial sets on $\mathcal{C}$ - reserving the symbol $\otimes$ for the standard action of $s$ Sets on the category $s \mathcal{C}$ of simplicial objects. We will refer to these as the internal and external actions respectively.

We can use the internal action to define a geometric realization functor for $s \mathcal{C}$. 
Definition 5.7. Let $\mathcal{C}$ be a simplicial model category and let $X \in s \mathcal{C}$ be a simplicial object in $\mathcal{C}$. Define the realization $|X| \in \mathcal{C}$ of $X$ by the coequalizer diagram in $\mathcal{C}$.

$$
\coprod_{\phi:[m] \rightarrow[n]} \Delta^{m} \otimes_{\mathcal{C}} X_{n} \longrightarrow \coprod_{n} \Delta^{n} \otimes_{\mathcal{C}} X_{n} \longrightarrow|X| .
$$

Here $\phi$ runs over the morphisms in $\Delta$ and the parallel arrows are obtained by evaluating on $\Delta^{(-)}$and $X_{(-)}$respectively.

EXAMPLES 5.8. 1.) This geometric realization functor from simplicial sets to CGH of Definition 1.19 can be obtained by regarding a simplicial set as a discrete simplicial space and applying the geometric realization functor $|-|: s \mathbf{C G H} \rightarrow$ CGH.

2.) Here is an Eilenberg-Zilber Theorem. If $X \in s s$ Sets, then there is a natural isomorphism $|X| \rightarrow \operatorname{diag}(X)$ where $\operatorname{diag}(X)$ is the diagonal simplicial set of the bisimplicial set $X$. To see this recall that the $k$-simplices of $\Delta^{n}$ are the morphisms $\phi:[k] \rightarrow[n]$ in $\Delta$. If $X=\left\{X_{n}\right\}$ is a simplicial object in simplicial sets, we can define

$$
\left(\Delta^{n} \times X_{n}\right)_{k}=\left(\Delta^{n}\right)_{k} \times\left(X_{n}\right)_{k} \rightarrow\left(X_{k}\right)_{k}
$$

sending $(\phi, y)$ to $\phi^{*}(y)$. It is an exercise to check that there is an induced map of simplicial sets

$$
\coprod_{n} \Delta^{n} \times X_{n} \rightarrow \operatorname{diag}(X)
$$

and that this map coequalizes the diagram of Definition 5.7.

3.) Let $A \in \mathcal{C}$ and $K$ a simplicial set. Then using the external action of $s$ Sets on $s \mathcal{C}$ we get an object $K \otimes A \in s \mathcal{C}$. Then there is a natural isomorphism $|K \otimes A| \cong K \otimes_{\mathcal{C}} A$. The argument goes more or less as in the previous example and is left as an exercise.

The functor $|-|: s \mathcal{C} \rightarrow \mathcal{C}$ has a right adjoint

$$
A \mapsto\left\{A^{\Delta^{n}}\right\} \stackrel{\text { def }}{=} A^{\Delta}
$$

where $A^{K}$ is the exponential object internal to $\mathcal{C}$. Then we have the following variation on one of Reedy's main results.

THEOREM 5.9. Let $\mathcal{C}$ be a simplicial model category. The adjoint pair

$$
|-|: s \mathcal{C} \rightleftarrows \mathcal{C}:(-)^{\Delta}
$$

is a Quillen functor from $s \mathcal{C}$ to $\mathcal{C}$. In particular, realization preserves cofibrations and weak equivalences between cofibrant objects.

Rather than prove this result, let us examine how the skeleton filtration interacts with realization. If $X \in s \mathcal{C}$ there is an isomorphism colim $\left|\operatorname{sk}_{n} X\right| \cong|X|$ and, using Proposition 5.2 and Example 5.8.3 we have a push-out diagram

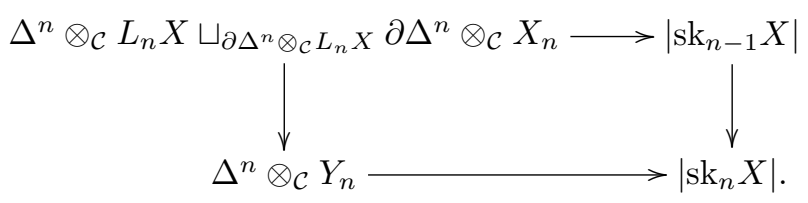


If $X$ is Reedy cofibrant, then the morphisms $L_{n} X \rightarrow X_{n}$ are cofibrations, and then the corner axiom for simplicial model categories (See Proposition 4.12) implies that $\left|\mathrm{sk}_{n-1} X\right| \rightarrow\left|\mathrm{sk}_{n} X\right|$ is a cofibration.

In the case when $X$ is $s$-free - which happens surprisingly often in applications - this push-out diagram specializes even more to a diagram that really looks like something you'd have with a simplicial complex. If $X$ is $s$-free on $\left\{Z_{k}\right\}$, then Proposition 5.3 yields a push-out diagram

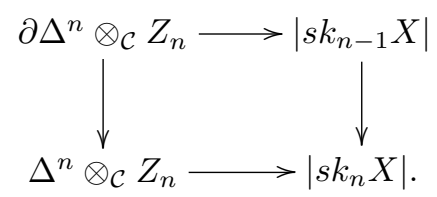

To specialize to an application, if $X \in s \mathbf{C G H}$ is a simplicial space, then the cofiber of $\left|\mathrm{sk}_{n-1} X\right| \rightarrow\left|\mathrm{sk}_{n} X\right|$ is the pointed space

$$
\left|\Delta^{n} / \partial \Delta^{n}\right| \wedge\left(X_{n} / L_{n} X\right) \cong S^{n} \wedge\left(X_{n} / L_{n} X\right)
$$

and we check that if $E_{*}$ is any generalized homology theory, then

$$
\tilde{E}_{p+q}\left(\left|\Delta^{p} / \partial \Delta^{p}\right| \wedge\left(X_{p} / L_{p} X\right)\right) \cong N \tilde{E}_{q} X_{p} .
$$

Thus it follows that there is a spectral sequence, for any Reedy cofibrant simplicial space $X$

$$
\pi_{p} E_{q}(X) \Longrightarrow E_{p+q}|X| .
$$

A variation of these ideas, using cosimplicial spaces and homotopy yields the extremely useful Bousfield-Kan spectral sequence of [7], which is the prototype of all second-quadrant homotopy spectral sequences.

5.3. Resolution model categories. The Reedy model category structure of the previous section has two drawbacks. First, our simplicial objects are often built as resolutions and, in so doing, we may want to specify the projective objects from which we build the resolutions. The Reedy model category structure doesn't do this - there are too many cofibrant objects. Second, after taking a resolution, we then would want to examine its realization, typically through some spectral sequence such as the homology spectral sequence of Equation 5.3. While a Reedy weak equivalence will certainly yield an isomorphism of spectral sequences, there are many other morphisms which also will yield an isomorphism of spectral sequences - there are not enough weak equivalences in $s \mathcal{C}$. We remedy both problems as once. The basic ideas go back to Dwyer, Kan, and Stover in [14] and [15], but they were greatly expanded in $[\mathbf{2 5}]$ and, especially, $[\mathbf{5}]^{6}$

For convenience and simplicity of language, we will work in a pointed model category $\mathcal{C}$ - that is, a category where the unique map from the initial object to terminal object is an isomorphism. Thus, for example, we will work with pointed spaces $\mathbf{C G H}_{*}$. We will also assume we have a simplicial model category. Both assumptions are unnecessary, as explained in [5], and can be removed at the cost of ramping up the language.

\footnotetext{
${ }^{6}$ Bousfield's paper is written cosimplicially, rather than simplicially, but the arguments are so categorical that they readily translate to the simplicial setting.
} 
If $A \in \mathcal{C}$ is cofibrant, the simplicial structure gives a canonical model for $\Sigma A$, the suspension of $A$ as the cofiber of

$$
\partial \Delta^{1} \otimes A \sqcup_{\partial \Delta^{1} \otimes *} \Delta^{1} \otimes * \longrightarrow \Delta^{1} \otimes A .
$$

Here $*$ is the initial (equals terminal) object. The cone, Cone $(A)$, on $A$ has a similar description.

We begin by specifying the building blocks of our resolutions.

Definition 5.10. Let $\mathcal{C}$ be our fixed simplicial model category and let $\mathbf{H o}(\mathcal{C})$ denote the homotopy category. Then a homotopy cogroup object $A \in \mathcal{C}$ is a cofibrant object so that $\operatorname{Ho}(\mathcal{C})(A,-)$ is a functor to groups. A set of projectives in $\mathcal{C}$ will be a set of homotopy cogroup objects which is closed under finite coproducts and suspension.

EXAmPles 5.11. 1. In $\mathbf{C G H}_{*}$, any suspension is a homotopy cogroup object. Indeed, if $X$ is any pointed CW-complex, we get a set of projectives by taking finite wedges of the spaces $\Sigma^{n} X, n \geq 1$. In particular, the example of [14] is given by setting $X=S^{0}$, whence the set of projectives is finite wedges of positive spheres.

2. A rich class of examples arises in spectra by considering homology theories for which there is a universal coefficient spectral sequence. See [19]. Spectra have not been discussed here, but there is a very rich literature. For a relatively straightforward model category on spectra whose homotopy category is the stable category, see $[6]$.

3. Let $\mathcal{C}=s \operatorname{Mod}_{R}$ for a commutative ring $R$. Then any cofibrant object is a homotopy cogroup object. An obvious set of projectives is given by taking finite sums of objects of the form $\Sigma^{n} R$ where $R$ is regarded as a constant simplicial $R$-module. Note $\Sigma^{n} R \cong K(R, n)$.

4. The category $s \mathbf{A} \mathbf{l g}_{R}$ is not pointed - and this is an example where we might want the unpointed generalization of this discussion here. In any case, if $M \in s \operatorname{Mod}_{R}$ is any simplicial cofibrant simplicial $R$-module, then the symmetric algebra $S_{R}(M) \in s \mathbf{A l g}_{R}$ is a homotopy cogroup object, in the obvious sense, and we might want to consider the set of projectives generated by $S_{R}\left(\Sigma^{n} R\right)$.

The following ideas are meant to echo the idea of projectives and surjections introduced on the very first page of these notes.

Definition 5.12. Let $\mathcal{C}$ be a pointed simplicial model category and $\mathbf{H o}(\mathcal{C})$ its homotopy category. Fix a set $\mathcal{P}$ of projectives in $\mathcal{C}$. Write $[-,-]$ for $\operatorname{Ho}(\mathcal{C})(-,-)$.

(1) A morphism $p: X \rightarrow Y$ in $\mathbf{H o}(\mathcal{C})$ is $\mathcal{P}$-epi if $p_{*}:[P, X] \rightarrow[P, Y]$ is onto for each $P \in \mathcal{P}$.

(2) An object $A \in \mathbf{H o}(\mathcal{C})$ is $\mathcal{P}$-projective if

$$
p_{*}:[A, X] \longrightarrow[A, Y]
$$

is onto for all $\mathcal{P}$-epi maps.

(3) A morphism $A \rightarrow B$ in $\mathcal{C}$ is called a $\mathcal{P}$-projective cofibration if it has the left lifting property for all $\mathcal{P}$-epi fibrations in $\mathcal{C}$.

The classes of $\mathcal{P}$-epi maps and of $\mathcal{P}$-projective objects determine each other; furthermore, every object in $\mathcal{P}$ is $\mathcal{P}$-projective. Note however, that the class of $\mathcal{P}$-projectives is closed under arbitrary coproducts. The class of $\mathcal{P}$-projective cofibrations will be characterized below; see Lemma 5.16. 
Lemma 5.13. The category $\operatorname{Ho}(\mathcal{C})$ has enough $\mathcal{P}$-projectives; that is, for every object $X \in \mathbf{H o}(\mathcal{C})$ there is a $\mathcal{P}$-epi $Y \rightarrow X$ with $Y \mathcal{P}$-projective.

Proof. We can take

$$
Y=\amalg_{P \in \mathcal{P}}^{\amalg} \underset{f: P \rightarrow X}{\amalg} P
$$

where $f$ ranges over all maps $P \rightarrow X$ in $\mathbf{H o}(\mathcal{C})$.

We now come to the $\mathcal{P}$-resolution model category structure. Recall that a morphism $f: A \rightarrow B$ of simplicial abelian groups is a weak equivalence if $f_{*}$ : $\pi_{*} A \rightarrow \pi_{*} B$ is an isomorphism. Also $f: A \rightarrow B$ is a fibration if the induced map of normalized chain complexes $N f: N A \rightarrow N B$ is surjective in positive degrees. We haven't been explicit about this, but the same statements hold for simplicial groups. If $A$ is a simplicial group, not necessarily abelian, we can still form the normalized complex $N A$ as in Example 2.15. We easily check that a morphism $f: A \rightarrow B$ of simplicial groups is a fibration if and only if it $N f$ is surjective in positive degrees.

Definition 5.14. Let $\mathcal{C}$ be a pointed simplicial model category with a fixed set of projectives $\mathcal{P}$. Let $f: X \rightarrow Y$ be a morphism in the category $s \mathcal{C}$ of simplicial objects in $\mathcal{C}$. Then

(1) the map $f$ is a $\mathcal{P}$-equivalence if the induced morphism

$$
f_{*}:[P, X] \longrightarrow[P, Y]
$$

is a weak equivalence of simplicial groups for all $P \in \mathcal{P}$;

(2) the map $f$ is a $\mathcal{P}$-fibration if it is a Reedy fibration and $f_{*}:[P, X] \longrightarrow[P, Y]$ is a fibration of simplicial groups for all $P \in \mathcal{P}$;

(3) the map $f$ is a $\mathcal{P}$-cofibration if the induced maps

$$
X_{n} \sqcup_{L_{n} X} L_{n} Y \longrightarrow Y_{n}, \quad n \geq 0,
$$

are $\mathcal{P}$-projective cofibrations.

Then, of course, the theorem is as follows.

Theorem 5.15. With these definitions of $\mathcal{P}$-equivalence, $\mathcal{P}$-fibration, and $\mathcal{P}$ cofibration, the category sC becomes a simplicial model category.

The proof is given in [5]. We call this the $\mathcal{P}$-resolution model category structure. It is cofibrantly generated if $\mathcal{C}$ is cofibrantly generated and an object is $\mathcal{P}$-fibrant if and only if it is Reedy fibrant. Furthermore, any Reedy weak equivalence is a $\mathcal{P}$-equivalence and any $\mathcal{P}$-cofibration is a Reedy cfoibration. The next result gives a characterization of $\mathcal{P}$-cofibrations.

Define a morphism $X \rightarrow Y$ in the category $\mathcal{C}$ to be $\mathcal{P}$-free if it can be written as a composition

$$
X \stackrel{i}{\longrightarrow} X \amalg F \stackrel{q}{\longrightarrow} Y
$$

where $i$ is the inclusion of the summand, $F$ is cofibrant and $\mathcal{P}$-projective, and $q$ is an acyclic cofibration. The following is also in [5].

Lemma 5.16. A morphism $X \rightarrow Y$ in $\mathcal{C}$ is a $\mathcal{P}$-projective cofibration if and only if it is a retract of $\mathcal{P}$-free map. 
REMARK 5.17. In his paper that inaugurated this subject [34], Stover wrote down a very concrete model for the $\mathcal{P}$-cofibrant replacement in $s \mathcal{C}$, at least in the case where every object is fibrant. If $P \in \mathcal{P}$, let Cone $(P)$ denote the cone on $P$. For $A$ in $\mathcal{C}$, define $V(A)$ by the push-out diagram



where the coproducts are over morphisms in $\mathcal{C}$. There are natural maps $V(A) \rightarrow A$ and $V(A) \rightarrow V^{2}(A)$. The first is given by evaluation and the second by noticing that each summand in the pushout diagram defines a morphism $P \rightarrow V(A)$ (or Cone $(P) \rightarrow V(A))$. Thus, by iterating $V$, we obtain an augmented simplicial object $V_{\bullet}(A) \rightarrow A$. Stover proves that this is a $\mathcal{P}$-equivalence and that $V_{\bullet}(A)$ is $\mathcal{P}$-cofibrant. It is also easy to prove that there is a (non-natural) homotopy equivalence $V(A) \rightarrow W$, where $W$ is isomorphic to a coproduct of projectives in $\mathcal{P}$.

EXAMPLES 5.18. 1. In the case where $\mathcal{C}=\mathbf{C G H}_{*}$ and $\mathcal{P}$ is the collection of finite wedges of spheres, a cofibrant replacement $X \rightarrow A$ for a space $X$ is a "resolution" by spheres. The map $|X| \rightarrow A$ will be a weak equivalence if $A$ is pathconnected or, more generally, will be a weak equivalence from $|X|$ to the component of the basepoint of $A$. These simplicial resolutions were used by Stover to define a spectral sequence for studying the homotopy groups of a wedge.

2. If we take $\mathcal{P}$ to be the collection of finite wedges of spheres $S^{k}, k \geq n$, then $|X| \rightarrow A$ will be a model for the $(n-1)$ st connected cover of $A$.

3. In [19], we resolved spectra by finite CW spectra $P$ with the property that $E_{*} P$ was projective for some homology theory $E$. This was an enormous help in developing a spectral sequence for studying the homotopy type of mapping spaces of structured ring spectra.

4. One big impact of these resolutions is to realization problems. For example, if $A$ is a pointed space, then $\pi_{*} A$ has a great deal of structure coming from Whitehead products, compositions, etc. Writing down axioms for this structure yields the notion of a $\Pi$-algebra and one can ask: given a $\Pi$-algebra $\Lambda$, in there a space $A$ so that $\pi_{*} A \cong \Lambda$ ? In $[\mathbf{3}]$ we defined and studied the space of all such realizations, and gave a decomposition of that space as an inverse limit of a tower where the layers are governed by André-Quillen cohomology. A transportation of these ideas to [19] yielded solutions to similar moduli problems in stable homotopy theory. Indeed, one statement of the celebrated Hopkins-Miller theorem is that the moduli space of all $E_{\infty}$-ring structures on Morava's $E_{n}$ spectrum is a space of the form $B G$, where $G$ is a group - in fact, the Morava stabilizer group. The basic algebraic fact at work is that $\left(E_{n}\right)_{*} \rightarrow\left(E_{n}\right)_{*} E_{n}$ is formally étale and, hence, has vanishing André-Quillen cohomology.

EXAMPLE 5.19. We meet a precursor of resolution model categories in Quillen's original work. See [30] $\S$ II.6. If $M$ and $N$ are two simplicial $R$-modules, let us define $M \otimes_{R}^{L} N$ to be the total left derived functor of $M \otimes_{R}(-)$ applied to $N$; that is, up to weak equivalence $M \otimes_{R}^{L} N=M \otimes_{R}^{L} Q$ where $Q$ is a cofibrant replacement for $N$. In [30], Quillen writes down a spectral sequence

$$
\operatorname{Tor}_{p}^{R}\left(\pi_{*} M, \pi_{*} N\right)_{q} \Longrightarrow \pi_{p+q}\left(M \otimes_{R}^{L} N\right) .
$$


The grading $q$ arises from the grading on the homotopy groups. Quillen's argument is essentially as follows.

Consider the resolution model category structure on $s\left(s \operatorname{Mod}_{R}\right)$ defined by the homotopy cogroup objects $\Sigma^{n} R=K(R, n)$. See Example 5.11.3. Then if we regard $N \in s \operatorname{Mod}_{R}$ as a constant simplicial object in $s\left(s \operatorname{Mod}_{R}\right)$, we can form a $\mathcal{P}$-cofibrant replacement $P_{\bullet} \rightarrow N$ for $N$. Taking homotopy in the old (internal) simplicial degree first, then in the new (external) simplicial degree, we have that $\pi_{0} \pi_{*} P_{\bullet} \cong \pi_{*} N$ and $\pi_{p} \pi_{*} P_{\bullet}=0$. Furthermore, $P_{p}$ is a cofibrant simplicial module homotopy equivalent to a direct sum of simplicial modules of the form $K(R, n)$. Together, these two facts imply that $Q=\operatorname{diag} P \bullet \rightarrow N$ is a cofibrant replacement.

To get the spectral sequence, form the bisimplicial module $M \otimes_{R} P_{\bullet}=\left\{M \otimes_{R}\right.$ $\left.P_{p}\right\}$ and filter by the new (external) simplicial degree $p$. Then the $E^{1}$ term is given by

$$
E_{p, q}^{1}=\pi_{p}\left(M \otimes_{R} \pi_{*} P_{p}\right)
$$

and we have, from the previous paragraph, that $\pi_{*} P \bullet \rightarrow \pi_{*} N$ is a graded projective resolution. So Quillen's spectral sequence follows.

\section{References}

[1] Basterra, M. and Mandell, M. "Homology and cohomology of $E_{\infty}$ ring spectra", Math. Z., 249 (2005), No. 4, 903-944.

[2] Berger, C. and Moerdijk, I., "Axiomatic homotopy theory for operads", Comment. Math. Helv., 78 (2003), No. 4, 805-831.

[3] Blanc, D. and Dwyer, W. G. and Goerss, P. G., "The realization space of a П-algebra: a moduli problem in algebraic topology, Topology, 43 (2004) No. 4, 857-892.

[4] Bousfield, A. K., "Homotopy spectral sequences and obstructions", Isr. J. Math. 66 (1989) $54-104$.

[5] Bousfield, A. K., "Cosimplicial resolutions and homotopy spectral sequences in model categories", Geom. Topol., 7 (2003), 1001-1053 (electronic).

[6] Bousfield, A. K. and Friedlander, E. M., "Homotopy theory of $\Gamma$-spaces, spectra, and bisimplicial sets", Geometric applications of homotopy theory (Proc. Conf., Evanston, Ill., 1977), II, Lecture Notes in Math, 658, 80-130, Springer-Verlag, Berlin 1978.

[7] Bousfield, A. K. and Kan, D. M., Homotopy limits, completions, and localizations, Lecture Notes in Math. 304 ( $2^{\text {nd }}$ corrected printing), Springer-Verlag, Berlin-Heidelberg-New York, 1987.

[8] Curtis, E.B., "Simplicial homotopy theory", Advances in Math., 6 (1971), 107-209.

[9] Dold, A., "Homology of symmetric products and other functors of complexes", Ann. of Math. (2), 68 (1958), 54-80.

[10] Dold, A. and Puppe, D., "Homologie nicht-additiver Funktoren. Anwendungen", Ann. Inst. Fourier Grenoble, 11 (1961), 201-312.

[11] Dwyer, W. G. and Kan, D. M., "Calculating simplicial localizations", J. Pure Appl. Algebra, 18 (1980), No. 1, 17-35.

[12] Dwyer, W. G. and Kan, D. M., "A classification theorem for diagrams of simplicial sets", Topology, 23 (1984) No.2, 139-155.

[13] Dwyer, W. G. and Kan, D. M., "Function complexes in homotopical algebra" Topology, 18 (1980), No. 4, 427-440.

[14] Dwyer, W. G., Kan, D. M., and Stover, C. R., " $E_{2}$ model category structure for pointed simplicial spaces," J. of Pure and Applied Algebra 90 (1993), 137-152.

[15] Dwyer, W. G., Kan, D. M., and Stover, C. R., "The bigraded homotopy groups $\pi_{i, j} X$ of a pointed simplicial space X", J. of Pure and Applied Algebra 103 (1995), 167-188.

[16] Dwyer, W. G. and Spaliński, J., "Homotopy theories and model categories", Handbook of algebraic topology, 73-126, North-Holland, Amsterdam, 1995.

[17] Gabriel, P. and Zisman, M., Calculus of fractions and homotopy theory, Ergebnisse der Mathematik und ihrer Grenzgebiete, Band 35, Springer-Verlag New York, Inc., New York. 1967. 
[18] Goerss, P. G., "André-Quillen cohomology and the homotopy groups of mapping spaces: understanding the $E_{2}$ term of the Bousfield-Kan spectral sequence," J. of Pure and Applied Algebra 63 (1990), pp. 113-153.

[19] Goerss, P. G. and Hopkins, M. J., "Moduli spaces of commutative ring spectra", Structured Ring Spectra, London Math. Soc. Lecture Note Ser. 315, 151-200, Cambridge Univ. Press, Cambridge, 2004.

[20] Goerss, P. G. and Jardine, J. F., Simplicial homotopy theory, Progress in Mathematics 174, Birkhäuser Verlag, Basel, 1999.

[21] Hirschhorn, P., Model categories and their localizations, Mathematical Surveys and Monographs, 99, American Mathematical Society, Providence, RI, 2002.

[22] Hovey, M., Model categories, Mathematical Surveys and Monographs, 63, American Mathematical Society, Providence, RI, 1999.

[23] Illusie, L,, Complexe cotangent et déformations. I, Lecture Notes in Mathematics, Vol. 239, Springer-Verlag, Berlin, 1971.

[24] Iyengar, S. "André-Quillen homology of commutative algebras", to appear.

[25] Jardine, J. F., "Bousfield's $E_{2}$ model theory for simplicial objects", Homotopy theory: relations with algebraic geometry, group cohomology, and algebraic K-theory, Contemp. Math., 346, 305-319, Amer. Math. Soc., Providence, RI, 2004.

[26] Kan, D. M., "A combinatorial definition of homotopy groups", Ann. of Math. (2), 67 (1958), $282-312$.

[27] Lewis, L.G., Jr, "The stable category and generalized Thom spectra", Thesis, University of Chicago, 1978.

[28] May, J. P., Simplicial objects in algebraic topology, Van Nostrand Mathematical Studies, No. 11, D. Van Nostrand Co., Inc., Princeton, N.J.-Toronto, Ont.-London, 1967.

[29] Quillen, D.G., "On the (co)-homology of commutative rings", Proc. Symp. Pure Math. 17 (1970), 65-87.

[30] Quillen D.G., Homotopical Algebra, Lecture Notes in Math. 43, Springer-Verlag, BerlinHeidelberg-New York, 1967.

[31] Quillen, D.G., "Rational homotopy theory", Ann. of Math. (2), 90 (1969), 205-295.

[32] Reedy, C. L., "Homotopy theory of model categories", Preprint, 1973. Available from http://math.mit.edu/ psh.

[33] Steenrod, N. E., "A convenient category of topological spaces", Michigan Math. J., 14 (1967), $133-152$.

[34] Stover, C. R., "A Van Kampen spectral sequence for higher homotopy groups," Topology 29 (1990), 9-26.

[35] Strøm, A., "The homotopy category is a homotopy category", Arch. Math. (Basel), 23 (1972), $435-441$.

Department of Mathematics, Northwestern University, Evanston IL 60208

E-mail address: pgoerss@math.northwestern.edu

Department of Mathematics \& Computer Science, Albion College, Albion, Mi 49224

E-mail address: kschemmerhorn@dom.edu

Current address: Dominican University, River Forest, IL 60305 